\title{
Rates in almost sure invariance principle for quickly mixing dynamical systems
}

\author{
C. Cuny*, J. Dedecker; A. Korepanov ${ }^{\ddagger}$ Florence Merlevède ${ }^{\S}$
}

21 November 2018

\begin{abstract}
For a large class of quickly mixing dynamical systems, we prove that the error in the almost sure approximation with a Brownian motion is of order $O\left((\log n)^{a}\right)$ with $a \geq 2$. Specifically, we consider nonuniformly expanding maps with exponential and stretched exponential decay of correlations, with one-dimensional Hölder continuous observables.
\end{abstract}

Keywords: Strong invariance principle, KMT approximation, Nonuniformly expanding dynamical systems, Markov chain.

$M S C:$ 60F17, 37E05.

\section{Introduction and main result}

Let $X$ be a bounded metric space and $f: X \rightarrow X$ be a transformation, preserving a Borel probability measure $\mu$. Suppose that $\varphi: X \rightarrow \mathbb{R}$ is Hölder continuous with $\int \varphi d \mu=0$. We consider the Birkhoff sums

$$
S_{n}(\varphi)=\sum_{k=0}^{n-1} \varphi \circ f^{k}
$$

as a discrete time random process, defined on the probability space $(X, \mu)$. It is common in chaotic dynamical systems that $S_{n}(\varphi)$ behaves like a Brownian motion. For example, $S_{n}(\varphi)$ may satisfy Donsker's invariance principle: the normalized process $X_{t}=n^{-1 / 2} S_{\lfloor n t\rfloor}(\varphi)$ may converge weakly to a Brownian motion as $n \rightarrow \infty$. A basic and natural question is, how close is $S_{n}(\varphi)$ to a Brownian motion? For many chaotic dynamical systems, $S_{n}(\varphi)$ can be almost surely approximated by a Brownian motion. In this work we look at the error of such approximations.

Definition 1.1. We say that a random process $\left(X_{n}\right)_{n \geq 0}$ satisfies the almost sure invariance principle (ASIP) with rate $o\left(r_{n}\right)$, where $r_{n}$ is a deterministic sequence such that $r_{n}=$ $o\left(n^{1 / 2} \log \log n\right)$, if, possibly enlarging the probability space, there exists a Brownian motion $W_{t}$ such that

$$
X_{n}=W_{n}+o\left(r_{n}\right) \quad \text { almost surely. }
$$

\footnotetext{
${ }^{*}$ Université de Brest, LMBA, UMR CNRS 6205. Email: christophe.cuny@univ-brest.fr

${ }^{\dagger}$ Université Paris Descartes, Sorbonne Paris Cité, Laboratoire MAP5 (UMR 8145). Email: jerome.dedecker@parisdescartes.fr

${ }^{\ddagger}$ University of Exeter, UK. Email: a.korepanov@exeter.ac.uk

${ }^{\S}$ Université Paris-Est, LAMA (UMR 8050), UPEM, CNRS, UPEC. Email: florence.merlevede@u-pem.fr
} 
The ASIP was introduced by Strassen [28] as a tool to prove the functional law of iterated logarithm. Besides that, the ASIP implies Donsker's invariance principle and a range of other laws, see for example Philipp and Stout 23].

A question of particular interest and challenge is to identify the optimal rate in the ASIP. Strassen conjectured that under the best realistic assumptions (such as independent increments assuming values $\{-1,+1\}$ with probability $1 / 2$ each), the best possible rate is $O\left(n^{1 / 4}(\log n)^{1 / 2}(\log \log n)^{1 / 4}\right)$. Kiefer [12] showed that, for martingales, this is the best rate one can obtain by mean of the Skorokhod embedding method. On the other hand, better rates were proved possible by Csörgő and Révész [6] and then by Komlós, Major and Tusnády [13] and Major [19]:

Theorem 1.2 (KMT approximation). Suppose that $X_{n}=\sum_{k=1}^{n} \xi_{k}$, where $\left(\xi_{k}\right)$ is a sequence of real valued independent and identically distributed random variables with $\mathbb{E} \xi_{1}=0$. Then

(a) if $\mathbb{E}\left(\left|X_{1}\right|^{p}\right)<\infty$ with $p>2$, then $\left(X_{n}\right)$ satisfies the ASIP with rate o $\left(n^{1 / p}\right)$;

(b) if $\mathbb{E}\left(e^{\delta\left|X_{1}\right|}\right)<\infty$ with $\delta>0$, then $\left(X_{n}\right)$ satisfies the ASIP with rate $O(\log n)$.

For processes with dependent increments, it took several decades to obtain better rates than $O\left(n^{1 / 4}(\log n)^{1 / 2}(\log \log n)^{1 / 4}\right)$. A number of different ways to establish the ASIP were found, but those with good rates required independence of increments and proved very hard to extend.

Recently the rate $o\left(n^{\varepsilon}\right)$ for arbitrarily small $\varepsilon>0$ was reached by Berkes, Liu and $\mathrm{Wu}[3]$ for processes driven by a Bernoulli shift:

$$
X_{n}=\sum_{k=1}^{n} \psi\left(\ldots, \xi_{k-1}, \xi_{k}, \xi_{k+1}, \ldots\right),
$$

where $\left(\xi_{k}\right)$ are independent and identically distributed (iid) random variables, and $\psi$ satisfies certain regularity assumptions. Then, Merlevède and Rio 21 obtained the rate $O(\log n)$ for processes of the type

$$
X_{n}=\sum_{k=1}^{n} \psi\left(g_{k}\right),
$$

where $\left\{g_{k}\right\}_{k \geq 1}$ is a geometrically ergodic Markov chain and $\psi$ is bounded.

In this paper we suppose that $f$ is a nonuniformly expanding map in the sense of Young [31, and $\mu$ is its unique physical (Sinai-Ruelle-Bowen) invariant measure. This covers, for example:

- uniformly expanding maps such as the doubling map, Gauss continued fraction map, $\beta$-shifts and Gibbs-Markov maps with onto branches;

- maps with critical points or indifferent fixed points, such as intermittent (PomeauManneville) maps [18, logistic maps with Collet-Eckmann parameters [5, 31] or AlvesViana maps [11];

- factors of nonuniformly hyperbolic maps, e.g. the collision map for dispersing billiards or Hénon map, which are instrumental for proving limit theorems including the ASIP [20].

We state the technical assumptions on the maps to which our results apply in Subsection 2.1.

Statistical properties of nonuniformly expanding maps are often proved using a suitable inducing scheme. One chooses a base $Y \subset X$ and a return time $\tau: Y \rightarrow \mathbb{N}$ with $f^{\tau(y)}(y) \in Y$ for all $y \in Y$, so that the induced map $f^{\tau}: Y \rightarrow Y$ is particularly nice, namely full branch Gibbs-Markov. 
An inducing scheme comes with a natural "reference" probability measure $m$ on $Y$ (e.g. Lebesgue), and the asymptotics of $m(\tau>n)$ as $n \rightarrow \infty$ largely determine what statistical properties one can prove. For example, if $f$ is mixing (more precisely, $\operatorname{gcd}\{\tau(y): y \in Y\}=1$ ), then the asymptotics of $m(\tau>n)$ give a useful bound on the covariances between the summands in $S_{n}(\varphi)$ [10, 15, 31]:

- if $m(\tau \geq n)=O\left(n^{-\beta}\right)$ with $\beta>1$, then $\operatorname{Cov}\left(\varphi, \varphi \circ f^{n}\right)=O\left(n^{-(\beta-1)}\right)$;

- if $m(\tau \geq n)=O\left(\mathrm{e}^{-A n^{\gamma}}\right)$ with $A>0$ and $0<\gamma \leq 1$, then $\operatorname{Cov}\left(\varphi, \varphi \circ f^{n}\right)=O\left(\mathrm{e}^{-B n^{\gamma}}\right)$ with some $B>0$.

(Recall that the probability space is $(X, \mu)$.)

Melbourne and Nicol [20] proved the ASIP for $S_{n}(\varphi)$ provided that $\tau \in L^{p}(m), p>2$. Their rates are of the type $o\left(n^{\varepsilon}\right)$, where $\varepsilon \in(1 / 4,1 / 2)$ depends on $p$.

Remark 1.3. The variance of the Brownian motion in the ASIP for $S_{n}(\varphi)$ is

$$
c^{2}=\lim _{n \rightarrow \infty} \frac{1}{n} \int\left|S_{n}(\varphi)\right|^{2} d \mu .
$$

For nonuniformly expanding maps with $\tau \in L^{2}(m)$, the limit exists by e.g. [16, Cor. 5.5].

Korepanov [14] applied the result of [3] to nonuniformly expanding dynamical systems, showing the ASIP with rate $o\left(n^{\varepsilon}\right)$ for every $\varepsilon>0$ under the assumption of exponential tails of the return times, i.e. $m(\tau>n)=O\left(\mathrm{e}^{-c n}\right)$ with $c>0$.

The method of [14] only works for exponential decay of return times. It was improved by the authors of this paper in [7], where we obtained a significantly more general result which covers maps with polynomial decay of return times, such as intermittent maps:

Theorem 1.4 ([7]). Let $f: X \rightarrow X$ be a nonuniformly expanding map (see Section [2.1) with the reference measure $m$, return time $\tau$ and physical invariant measure $\mu$. Let $\varphi: X \rightarrow \mathbb{R}$ be a Hölder continuous function with $\int \varphi d \mu=0$. Consider the random process $S_{n}(\varphi)=\sum_{k=0}^{n-1} \varphi \circ f^{k}$ on the probability space $(X, \mu)$.

(a) If $m(\tau>n)=O\left(n^{-\beta}\right)$ with $\beta>2$, then the process $\left(S_{n}(\varphi)\right)_{n \geq 0}$ satisfies the ASIP with the rate $o\left(n^{1 / \beta}(\log n)^{1 / \beta+\varepsilon}\right)$ for every $\varepsilon>0$.

(b) If $\int \tau^{\beta} d m<\infty$ with $\beta>2$, then the process $\left(S_{n}(\varphi)\right)_{n \geq 0}$ satisfies the ASIP with the rate $o\left(n^{1 / \beta}\right)$.

Remark 1.5. The rate in Theorem 1.4 (a) is essentially optimal: $\varepsilon$ cannot be reduced to 0 , for example, for the intermittent maps.

When the return times decay faster than polynomially, Theorem 1.4 gives the ASIP with rate $o\left(n^{\varepsilon}\right)$ for any $\varepsilon>0$, the same as in [14]. This, however, is suboptimal in case of exponential and stretched exponential tails. We fix this in the present paper. Our main result is:

Theorem 1.6. Let $f: X \rightarrow X$ be a nonuniformly expanding map (see Section 2.1) with the reference measure $m$, return time $\tau$ and physical invariant measure $\mu$. Let $\varphi: X \rightarrow \mathbb{R}$ be a Hölder continuous function with $\int \varphi d \mu=0$. Consider the random process $S_{n}(\varphi)=\sum_{k=0}^{n-1} \varphi \circ f^{k}$ on the probability space $(X, \mu)$.

If $m(\tau>n)=O\left(\mathrm{e}^{-\kappa n^{\gamma}}\right)$ with $\kappa>0$ and $\left.\left.\gamma \in\right] 0,1\right]$, then the process $\left(S_{n}(\varphi)\right)_{n \geq 0}$ satisfies the ASIP with the rate $O\left((\log n)^{1+1 / \gamma}\right)$. 
Remark 1.7. For the doubling map, Theorem 1.6 gives the rate $O\left((\log n)^{2}\right)$, improving the rate $o\left(n^{\varepsilon}\right)$ for every $\varepsilon>0$ in $[3$. We conjecture that it can be further improved to $O(\log n)$, which is known to be optimal for processes with independent and identically distributed increments [13] and additive bounded functionals of geometrically ergodic Markov chains [21. We expect the same for all maps which are nonuniformly expanding with exponential decay of return times: our rate $O\left((\log n)^{2}\right)$ should be eventually reduced to $O(\log n)$.

Remark 1.8. In most examples of nonuniformly expanding maps, the return times decay exponentially, except the intermittent maps with polynomial decay and Alves-Viana maps where the best available estimates are stretched exponential, namely $\mathrm{e}^{-c \sqrt{n}}$, see [2, 11]. To enhance our portfolio of examples, in Appendix $\mathrm{A}$ we present a family of interval maps with decay $O\left(\mathrm{e}^{-c n^{\gamma}}\right)$, parametrized by $\gamma \in] 0,1]$.

Remark 1.9. In a class of nonuniformly hyperbolic dynamical systems, the ASIP can be deduced from the corresponding result on a nonuniformly expanding map, with the same rate, using the so-called Sinai trick [20. For example, for dispersing billiards and Hénon maps, where the return times have exponential tails, we obtain the rate $O\left((\log n)^{2}\right)$.

The paper is organized as follows. In Section 2, we give a formal definition of the class of nonuniformly expanding maps to which our results apply. Further, we redefine the random process $S_{n}(\varphi)$ on a Markov shift without changing its distribution. In Section 3, we prove Theorem 1.6. The proof is based on the construction of approximating Brownian motion from [3] as in [8] and [7. One of the crucial tools is the ASIP for processes with independent (but not necessarily identically distributed) increments by Sakhanenko [25, Thm. 1]. Sakhanenko's result generalizes KMT's result (concerning iid random variables having a finite moment generating function in a neighborhood of 0 ) to non-identically distributed random variables whose distributions satisfy a condition equivalent to the condition in Bernstein's well-known inequality (as shown by Zaitsev [32]).

Throughout the paper, we shall often use the notation $a_{n} \ll b_{n}$ which means that there exists a universal constant $C$ such that, for all $n \geq 1, a_{n} \leq C b_{n}$.

\section{Reduction to a Markov shift}

\subsection{Nonuniformly expanding maps}

Here we state formal assumptions on dynamical systems, to which our results apply. Briefly, we require that they admit a Young tower, i.e. an inducing scheme with a full branch Gibbs-Markov base map and certain regularity assumptions. Often Young towers are difficult to construct, but they provide a universal framework for proving limit theorems. For uniformly expanding maps, including those that are not Markov or conformal, one could verify the general assumptions of Eslami [9, Sec. 7.2].

Let $X$ be a complete bounded separable metric space with the Borel $\sigma$-algebra. Suppose that $f: X \rightarrow X$ is a measurable transformation which admits an inducing scheme consisting of:

- a closed subset $Y$ of $X$ with a reference probability measure $m$ on $Y$;

- a finite or countable partition $\alpha$ of $Y$ (up to a zero measure set) with $m(a)>0$ for all $a \in \alpha$

- an integrable return time function $\tau: Y \rightarrow\{1,2, \ldots\}$ which is constant on each $a \in \alpha$ with value $\tau(a)$ and $f^{\tau(a)}(y) \in Y$ for all $y \in a, a \in \alpha$. (We do not require that $\tau$ is the first return time to $Y$.) 
Let $F: Y \rightarrow Y, F(y)=f^{\tau(y)}(y)$ be the induced map. We assume that there are constants $\kappa>1, K>0$ and $\eta \in(0,1]$ such that for each $a \in \alpha$ and all $x, y \in a$ :

- $F$ restricts to a (measure-theoretic) bijection from $a$ to $Y$;

- $d(F(x), F(y)) \geq \kappa d(x, y)$;

- $d\left(f^{k}(x), f^{k}(y)\right) \leq K d(F(x), F(y))$ for all $0 \leq k \leq \tau(a)$;

- the inverse Jacobian $\zeta_{a}=\frac{d m}{d m \circ F}$ of the restriction $F: a \rightarrow Y$ satisfies

$$
|\log | \zeta_{a}(x)|-\log | \zeta_{a}(y)|| \leq K d(F(x), F(y))^{\eta} .
$$

In addition to the standard assumptions above, we rely on non-pathological coding of orbits under $F$ allow by the elements of $\alpha$. Let $\mathcal{A}$ be the set of all finite words in the alphabet $\alpha$ and $Y_{w}=\cap_{k=0}^{n} F^{-k}\left(a_{k}\right)$ for $w=a_{0} \cdots a_{n} \in \mathcal{A}$. We require that

$$
m\left(Y_{w}\right)=m\left(\bar{Y}_{w}\right) \quad \text { for every } w \in \mathcal{A} .
$$

We say that the map $f$ as above is nonuniformly expanding. We refer to $F: Y \rightarrow Y$, $F(x)=f^{\tau(x)}(x)$ as the induced map. It is standard [1, Cor. p. 199], [31, Proof of Thm. 1] that there is a unique absolutely continuous $F$-invariant probability measure $\mu_{Y}$ on $Y$ with $\frac{1}{c} \leq d \mu_{Y} / d m \leq c$ for some $c>0$, and the corresponding $f$-invariant probability measure $\mu$ on $X$.

We say that the return times of $f$ have:

- a weak polynomial moment of order $\beta \geq 1$, if $m(\tau \geq n) \ll n^{-\beta}$;

- a strong polynomial moment of order $\beta \geq 1$, if $\int \tau^{\beta} d m<\infty$;

- a subexponential moment of order $\gamma \in] 0,1]$, if $\int \mathrm{e}^{\delta \tau^{\gamma}} d m<\infty$ for some $\delta>0$.

\subsection{Markov shift}

Following [7, 14], for nonuniformly expanding dynamical systems, the random process $S_{n}(\varphi)$ can be redefined on a Markov shift without changing its distribution. The structure of the Markov shift is as follows.

Let $\left(\mathcal{A}, \mathbb{P}_{\mathcal{A}}\right)$ be a countable probability space and $h: \mathcal{A} \rightarrow\{1,2, \ldots\}$ with $\mathbb{E}_{\mathcal{A}}(h)<\infty$. Let

$$
S=\{(w, \ell) \in \mathcal{A} \times \mathbb{Z}: 0 \leq \ell<h(w)\} .
$$

We construct a stationary Markov chain $g_{0}, g_{1}, \ldots$ on $S$ such that if $g_{n}=(w, \ell)$ with $\ell<$ $h(w)-1$, then $g_{n+1}=(w, \ell+1)$, while if $g_{n}=(w, \ell)$ with $\ell=h(w)-1$, then $g_{n+1}=\left(w^{\prime}, 0\right)$, with $w^{\prime} \sim \mathbb{P}_{\mathcal{A}}$ independent from $\left(g_{k}\right)_{k \leq n}$.

The stationary measure of our Markov chain we denote by $\nu$. For $(w, \ell) \in S$,

$$
\nu(w, \ell)=\frac{\mathbb{P}_{\mathcal{A}}(\omega)}{\mathbb{E}_{\mathcal{A}}(h)} .
$$

It is convenient to represent $\left(g_{n}\right)_{n \geq 0}$ as generated by a sequence of independent innovations, as follows. Let $g_{0} \sim \nu$ and let $\varepsilon_{1}, \varepsilon_{2}, \ldots$ be a sequence of independent (also from $g_{0}$ ) and identically distributed random variables with values in $\mathcal{A}$ and distribution $\mathbb{P}_{\mathcal{A}}$. Let

$$
g_{n+1}=U\left(g_{n}, \varepsilon_{n+1}\right),
$$


where

$$
U((w, \ell), \varepsilon)= \begin{cases}(w, \ell+1), & \ell<h(w)-1, \\ (\varepsilon, 0), & \ell=h(w)-1 .\end{cases}
$$

Let $\Omega \subset S^{\mathbb{N}}$ be the space of possible trajectories of $\left(g_{n}\right)$ (i.e. sequences which correspond to non-zero probability transitions), and let $\mathbb{P}_{\Omega}$ be the corresponding probability measure. let

Let $\lambda>1$. For $a=\left(g_{0}, \ldots, g_{n}, g_{n+1}, \ldots\right)$ and $b=\left(g_{0}, \ldots, g_{n}, g_{n+1}^{\prime}, \ldots\right)$ with $g_{n+1} \neq g_{n+1}^{\prime}$,

$$
d(a, b)=\lambda^{-\#\left\{1 \leq k \leq n: g_{k} \in S_{0}\right\}},
$$

where $S_{0}=\{(w, \ell) \in S: \ell=0\}$. Then $d$ is a separation metric on $\Omega$, the separation time counted in terms of returns to $S_{0}$.

We proved [7, 14] that given a nonuniformly expanding dynamical system $f: X \rightarrow X$ with a Hölder continuous observable $\varphi: X \rightarrow \mathbb{R}$, there exists a Markov chain as above and a Hölder continuous function $\psi: \Omega \rightarrow \mathbb{R}$, such that

$$
\left\{\varphi \circ f^{n}\right\}_{n \geq 0} \stackrel{d}{=}\left\{\psi\left(g_{n}, g_{n+1}, \ldots\right)\right\}_{n \geq 0} .
$$

(The equality is in law, and the probability measures are $\mu$ and $\mathbb{P}_{\Omega}$ respectively.) Moreover, $h$ has essentially the same tails as $\tau$ :

- (weak polynomial moment) if $m(\tau \geq n) \ll n^{-\beta}$ with $\beta \geq 1$, then $\mathbb{P}_{\mathcal{A}}(h \geq n) \ll n^{-\beta}$;

- (strong polynomial moment) if $\int \tau^{\beta} d m<\infty$ with $\beta \geq 1$, then $\int h^{\beta} d \mathbb{P}_{\mathcal{A}}<\infty$;

- (subexponential moment) if $\int \mathrm{e}^{\delta \tau^{\gamma}} d m<\infty$ with $\left.\left.\gamma \in\right] 0,1\right]$ and $\delta>0$, then $\int \mathrm{e}^{\delta^{\prime} h^{\gamma}} d \mathbb{P}_{\mathcal{A}}<\infty$ with some $\delta^{\prime}>0$.

Denote

$$
X_{n}=\psi\left(g_{n}, g_{n+1}, \ldots\right) \quad \text { and } \quad S_{n}=\sum_{k=1}^{n} X_{k} .
$$

Thus, the ASIP for $S_{n}(\varphi)$ is reduced to the ASIP for $S_{n}$.

\section{$2.3 \quad$ Meeting time}

Following [7, Appendix A], for the purpose of proving the ASIP, we assume without loss of generality that $\operatorname{gcd}\{h(w): w \in \mathcal{A}\}=1$, that is the Markov chain $\left(g_{n}\right)$ is aperiodic.

Let $g_{0}^{*}$ be a random variable in $S$ with distribution $\nu$, independent from $g_{0}$ and $\left(\varepsilon_{n}\right)_{n \geq 1}$. Let $g_{0}^{*}, g_{1}^{*}, g_{2}^{*}, \ldots$ be a Markov chain given by

$$
g_{n+1}^{*}=U\left(g_{n}^{*}, \varepsilon_{n+1}\right) \text { for } n \geq 0 .
$$

Thus the chains $\left(g_{n}\right)_{n \geq 0}$ and $\left(g_{n}^{*}\right)_{n \geq 0}$ have independent initial states, but share the same innovations. Define the meeting time:

$$
T=\inf \left\{n \geq 0: g_{n}=g_{n}^{*}\right\} .
$$

Recall that $\mathbb{P}_{\mathcal{A}}(h \geq n)=O\left(\mathrm{e}^{-c n^{\gamma}}\right)$. This translates into a similar bound for $T$ :

Lemma 2.1. There exists $\delta>0$ such that $\mathbb{P}(T \geq n)=O\left(\mathrm{e}^{-\delta n^{\gamma}}\right)$.

The proof is omitted since it uses the same argument as in [7, Lemma 3.1], namely the result of Lindvall [17] (see also [24, Prop. 9.6]).

It is noteworthy to indicate that Lemma 2.1 implies the following control on the covariances: 
Lemma 2.2. There exists $\delta>0$ such that $\left|\operatorname{Cov}\left(X_{0}, X_{n}\right)\right|=O\left(\mathrm{e}^{-\delta n^{\gamma}}\right)$.

To prove the lemma above it suffices to follow the proof of Lemma 3.3 in [7] and to take into account Lemma 2.1 and Proposition 2.3 below whose proof is postponed to Appendix $\mathrm{B}$.

Proposition 2.3. Let $\delta_{n}: \Omega \rightarrow \mathbb{R}$,

$$
\delta_{n}\left(g_{0}, g_{1}, \ldots\right)=\sup \left|\psi\left(g_{0}, \ldots, g_{n}, g_{n+1}, g_{n+2}, \ldots\right)-\psi\left(g_{0}, \ldots, g_{n}, \tilde{g}_{n+1}, \tilde{g}_{n+2}, \ldots\right)\right|,
$$

where the supremum is taken over all possible $\left(\tilde{g}_{n+1}, \tilde{g}_{n+2}, \ldots\right)$. Then there exists $\delta>0$ such that

$$
\mathbb{E}\left(\delta_{n}\right)=O\left(\mathrm{e}^{-\delta n^{\gamma}}\right) .
$$

\section{Proof of Theorem 1.6}

Let $\alpha=1+\gamma^{-1}$. Our goal is to prove the ASIP for the random process $\left(S_{n}\right)$, driven by the stationary Markov chain $\left(g_{n}\right)$, as defined in Section 2 (see the definition (2.5)). Recall also that following [7, Appendix A], we can and do assume without loss of generality that the Markov chain $\left(g_{n}\right)$ is aperiodic.

The variance of the Brownian motion in the ASIP is, necessarily,

$$
c^{2}=\lim _{n \rightarrow \infty} \frac{1}{n} \int\left|S_{n}(\varphi)\right|^{2} d \mu=\lim _{n \rightarrow \infty} \frac{\mathbb{E}\left(S_{n}^{2}\right)}{n} .
$$

Technically, we prove the following strong approximation: one can redefine $\left(S_{n}\right)_{n \geq 1}$ without changing its distribution on a probability space (possibly richer than $\left(\Omega, \mathbb{P}_{\Omega}\right)$ ), on which there exists a sequence $\left(N_{i}\right)_{i \geq 1}$ of iid centered Gaussian r.v.'s with variance $c^{2}$ such that

$$
\sup _{k \leq n}\left|S_{n}-\sum_{i=1}^{k} N_{i}\right|=O\left((\log n)^{\alpha}\right) \quad \text { a.s. }
$$

Assume first that $c^{2}=0$. Note that, for any $\varepsilon>0$,

$$
\sum_{n \geq 1} m\left(\tau>\varepsilon(\log n)^{\alpha}\right)<\infty
$$

Therefore using the same arguments as those developed in the proof of Corollary 5.5 in [7], we can conclude that Theorem 1.6 holds with $c^{2}=0$.

Through the reminder of this section, we assume that $c^{2}>0$ and use the notation $b_{n}=$ $\lceil(\log n) /(\log 3)\rceil$ for $n \geq 2$ (so that $b_{n}$ is the unique integer such that $\left.3^{b_{n}-1}<n \leq 3^{b_{n}}\right)$. Let $\delta$ be the minimum of the constants $\delta$ involved in Lemmas 2.1 and 2.2 and Proposition 2.3. Fix $\kappa>0$ so that $\delta\left(2^{-1} \kappa\right)^{\gamma} \geq \log 3$. For $\ell \geq 0$, let

$$
m_{\ell}=\left[\kappa \ell^{1 / \gamma}\right] \vee 1 .
$$

Following [3], the proof of (3.1) it is divided into several steps. 


\section{$3.1 \quad$ Step 1}

Let

$$
X_{\ell, k}=\mathbb{E}_{g}\left(\psi\left(g_{k}, g_{k+1}, \ldots, g_{k+m_{\ell}},\left(\tilde{g}_{i}\right)_{i \geq k+m_{\ell}+1}\right)\right),
$$

where $\mathbb{E}_{g}$ denotes the conditional expectation given $g=\left(g_{n}\right)_{n \geq 0}$. Here $\left(\tilde{g}_{i}\right)_{i \geq k+m_{\ell}+1}$ is defined as follows: $\tilde{g}_{k+m_{\ell}+1}=U\left(g_{k+m_{\ell}}, \varepsilon_{k+m_{\ell}+1}^{\prime}\right)$ and $\tilde{g}_{i+1}=U\left(\tilde{g}_{i}, \varepsilon_{i+1}^{\prime}\right)$ for any $i>k+m_{\ell}$, where $\left(\varepsilon_{i}^{\prime}\right)_{i \geq 1}$ is an independent copy of $\left(\varepsilon_{i}\right)_{i \geq 1}$, independent of $g_{0}$, and $U$ is given by (2.3). Note that the $X_{\ell, k}$ 's are centered. Define

$$
W_{\ell, i}=\sum_{k=1+3^{\ell-1}}^{i+3^{\ell-1}} X_{k}, \quad \bar{W}_{\ell, i}=\sum_{k=1+3^{\ell-1}}^{i+3^{\ell-1}} X_{\ell, k} \quad \text { and } \quad W_{\ell, i}^{\prime}=W_{\ell, i}-\bar{W}_{\ell, i} .
$$

The fist step is to prove

Lemma 3.1.

$$
\sum_{\ell=1}^{b_{n}-1} W_{\ell, 3^{\ell}-3^{\ell-1}}^{\prime}+W_{b_{n}, n-3^{b_{n}-1}}^{\prime}=O\left((\log n)^{\alpha}\right) \quad \text { a.s. }
$$

Proof. By Proposition 2.3,

$$
\left\|\max _{1 \leq i \leq 3^{\ell}-3^{\ell-1}}\left|W_{k, \ell}^{\prime}\right|\right\|_{1} \leq \sum_{k=1+3^{\ell-1}}^{3^{\ell}}\left\|X_{k}-X_{\ell, k}\right\|_{1} \ll 3^{\ell} \exp \left(-\delta m_{\ell}^{\gamma}\right) \leq 3^{\ell} \exp \left(-\delta \times\left(2^{-1} \kappa\right)^{\gamma} \ell\right) .
$$

Using $\delta \times\left(2^{-1} \kappa\right)^{\gamma} \geq \log 3$,

$$
\sum_{\ell \geq 1} \ell^{-\alpha}\left\|\max _{1 \leq i \leq 3^{\ell}-3^{\ell-1}}\left|W_{k, \ell}^{\prime}\right|\right\|_{1}<\infty
$$

Now (3.3) follows from the Kronecker's lemma.

\subsection{Step 2.}

For $k \geq m_{\ell}+1$, let

$$
\tilde{X}_{\ell, k}=\mathbb{E}\left(X_{\ell, k} \mid \varepsilon_{k-m_{\ell}}, \ldots, \varepsilon_{k+m_{\ell}}\right) .
$$

Set $\ell_{0}=\inf \left\{\ell \geq 1: 3^{\ell-1} \geq \kappa \ell^{1 / \gamma}\right\}$. For $\ell \geq \ell_{0}$, define

$$
\widetilde{W}_{\ell, i}=\sum_{k=1+3^{\ell-1}}^{i+3^{\ell-1}} \tilde{X}_{\ell, k} \quad \text { and } \quad W_{\ell, i}^{\prime \prime}=\bar{W}_{\ell, i}-\widetilde{W}_{\ell, i}
$$

In the second step we prove

Lemma 3.2.

$$
\sum_{\ell=\ell_{0}}^{b_{n}-1} W_{\ell, 3^{\ell}-3^{\ell-1}}^{\prime \prime}+W_{b_{n}, n-3^{b_{n}-1}}^{\prime \prime}=O\left((\log n)^{\alpha}\right) \quad \text { a.s. }
$$

Proof. The result follows from the Kronecker's lemma, once we show that

$$
\sum_{\ell \geq \ell_{0}} \ell^{-\alpha} \sum_{k=3^{\ell-1}+1}^{3^{\ell}}\left\|X_{\ell, k}-\tilde{X}_{\ell, k}\right\|_{1}<\infty .
$$


By the estimate [7, (4.10)],

$$
\sum_{\ell \geq \ell_{0}} \ell^{-\alpha} \sum_{k=3^{\ell-1}+1}^{3^{\ell}}\left\|X_{\ell, k}-\tilde{X}_{\ell, k}\right\|_{1} \leq 2|\psi|_{\infty} \sum_{\ell \geq \ell_{0}} \frac{3^{\ell}}{\ell^{\alpha}} \mathbb{P}\left(T \geq m_{\ell}\right) .
$$

By Lemma 2.1, $\mathbb{P}(T \geq n)=O\left(\exp \left(-\delta n^{\gamma}\right)\right)$. Since $m_{\ell} \geq \kappa \ell^{1 / \gamma} / 2$ and $\delta\left(2^{-1} \kappa\right)^{\gamma} \geq \log 3$, the bounds (3.6) and (3.5) follow.

\subsection{Step 3. (Conditional Gaussian approximation)}

Set

$$
\tilde{S}_{n}=\sum_{\ell=\ell_{0}}^{b_{n}-1} \widetilde{W}_{\ell, 3^{\ell-3^{\ell-1}}}+\widetilde{W}_{b_{n}, n-3^{b_{n}-1}} .
$$

Let $K_{0}=\inf \left\{k \geq 1: m_{k} \leq 4^{-1} 3^{k-2}\right\}$. For $\ell \geq K_{0}$, let

$$
q_{\ell}=\left[3^{\ell-2} / m_{\ell}\right]-2 .
$$

Note that $q_{\ell} \rightarrow \infty$, as $\ell \rightarrow \infty$ and $q_{\ell} \geq 2$ whenever $\ell \geq K_{0}$. For $\ell \geq K_{0}$ and $j=1, \ldots, q_{\ell}$, set

$$
B_{\ell, j}=\sum_{i=1+(6 j-1) m_{\ell}}^{(6 j+5) m_{\ell}} \tilde{X}_{\ell, i+m_{\ell}+3^{\ell-1}} .
$$

Let $B_{\ell, j}=0$ if $\ell<K_{0}$. In what follows, we assume that $n \geq N_{0}=3^{K_{0}}$. Define

$$
S_{n}^{\diamond}=\sum_{\ell=K_{0}}^{b_{n}-1} \sum_{j=1}^{q_{\ell}} B_{\ell, j}+\sum_{j=1}^{\tau_{n}} B_{b_{n}, j}, \text { where } \tau_{n}=\left[\frac{n-3^{b_{n}-1}}{6 m_{b_{n}}}\right]-2 .
$$

Note that $\tau_{n} \leq q_{b_{n}}$. Moreover, since $\left\|\tilde{X}_{k, i}\right\|_{\infty} \leq|\psi|_{\infty}$ a.s., we infer that there exists a positive constant $C$ not depending on $n$ such that

$$
\max _{N_{0} \leq i \leq n}\left|\tilde{S}_{i}-S_{i}^{\diamond}\right| \leq C \sum_{k=1}^{b_{n}} m_{k}=O\left((\log n)^{\alpha}\right) \text { a.s. }
$$

Taking into account (3.3), (3.5) and (3.9), we see that (using for instance Lemma 4.1 of BerkesLiu-Wu [3] (3.1) is reduced to prove that one can redefine $\left(S_{n}^{\diamond}\right)_{n \geq 1}$ without changing its distribution on a (richer) probability space on which there exists iid random variables $\left(N_{i}\right)_{i \geq 1}$ with common distribution $\mathcal{N}\left(0, c^{2}\right)$, such that,

$$
S_{n}^{\diamond}-\sum_{i=1}^{n} N_{i}=O\left((\log n)^{\alpha}\right) \text { a.s. }
$$

where we recall that $S_{n}^{\diamond}$ is defined in (3.8). To prove this strong approximation result, we proceed as in steps 3.2 and 3.3 of the proof of Theorem 2.1 in Berkes-Liu-Wu [3]. Their step 3.2 consists in showing a conditional Gaussian approximation that is the object of our step 3. This requires several preliminary notations that we recall below for an easy understanding of the proof. With this aim, note first that for any integer $i \in\left[3^{\ell-1}+1,3^{\ell}\right]$ with $\ell \geq K_{0}$, we can write

$$
\tilde{X}_{\ell, i}=G_{\ell}\left(\varepsilon_{i-m_{\ell}}, \ldots, \varepsilon_{i+m_{\ell}}\right)
$$


where $G_{\ell}$ is a bounded measurable function. So $\tilde{X}_{\ell, i}$ is a bounded measurable function of $\left(\varepsilon_{i-m_{\ell}}, \ldots, \varepsilon_{i+m_{\ell}}\right)$.

Define, for $j \geq 1$,

$$
\begin{gathered}
\mathcal{J}_{\ell, j}=\left\{3^{\ell-1}+(6 j-1) m_{\ell}+k, k=1,2, \ldots, 2 m_{\ell}\right\}, \\
\boldsymbol{\eta}_{\ell, j}=\left(\varepsilon_{i}, i \in \mathcal{J}_{\ell, j}\right) \text { and } \boldsymbol{\eta}=\left(\boldsymbol{\eta}_{\ell, j}, j=1, \ldots, q_{\ell}+1\right)_{\ell=K_{0}}^{\infty} .
\end{gathered}
$$

Note that

$$
\begin{array}{r}
B_{\ell, j}=\sum_{i=1+(6 j-1) m_{\ell}}^{(6 j+1) m_{\ell}} \tilde{X}_{\ell, i+m_{\ell}+3^{\ell-1}}+\sum_{i=1+(6 j+1) m_{\ell}}^{(6 j+3) m_{\ell}} \tilde{X}_{\ell, i+m_{\ell}+3^{\ell-1}}+\sum_{i=1+(6 j+3) m_{\ell}}^{(6 j+5) m_{\ell}} \tilde{X}_{\ell, i+m_{\ell}+3^{\ell-1}} \\
:=H_{\ell}\left(\boldsymbol{\eta}_{\ell, j},\left\{\varepsilon_{i+3^{\ell-1}}\right\}_{1+(6 j+1) m_{\ell} \leq i \leq(6 j+5) m_{\ell}}, \boldsymbol{\eta}_{\ell, j+1}\right) .
\end{array}
$$

Let now $\left(u_{\ell}, \ell \in \mathbb{N}\right)$ be elements of $\mathcal{A}$ and set $\mathbf{u}=\left(\mathbf{u}_{k, j}, j=1, \ldots, q_{k}+1\right)_{k=K_{0}}^{\infty}$ where for any $j=1, \ldots, q_{k}+1, \mathbf{u}_{k, j}=\left(u_{\ell}, \ell \in \mathcal{J}_{k, j}\right)$. The idea is to use the fact that, on the set $\{\boldsymbol{\eta}=\mathbf{u}\}$, $\left(B_{\ell, j}(\mathbf{u})\right)_{j=1, \ldots, q_{\ell}}$ are independent between them. With this aim, define the following random functions: for $j \geq 1$,

$$
\begin{gathered}
F_{k, j}^{(1)}\left(\mathbf{u}_{k, j}\right)=\sum_{i=1+(6 j-1) m_{k}}^{(6 j+1) m_{k}} G_{k}\left(u_{i+3^{k-1}}, \ldots, u_{\left.(6 j+1) m_{k}+3^{k-1}, \varepsilon_{(6 j+1) m_{k}+1+3^{k-1}}, \ldots, \varepsilon_{i+2 m_{k}+3^{k-1}}\right),}\right. \\
F_{k, j}^{(2)}=\sum_{i=1+(6 j+1) m_{k}}^{(6 j+3) m_{k}} G_{k}\left(\varepsilon_{i+3^{k-1}}, \ldots, \varepsilon_{(6 j+1) m_{k}+3^{k-1}}, \varepsilon_{(6 j+1) m_{k}+1+3^{k-1}}, \ldots, \varepsilon_{i+2 m_{k}+3^{k-1}}\right),
\end{gathered}
$$

and

$F_{k, j}^{(3)}\left(\mathbf{u}_{k, j+1}\right)=\sum_{i=1+(6 j+3) m_{k}}^{(6 j+5) m_{k}} G_{k}\left(\varepsilon_{i+3^{k-1}}, \ldots, \varepsilon_{(6 j+5) m_{k}+3^{k-1}}, u_{(6 j+5) m_{k}+1+3^{k-1}}, \ldots, u_{i+2 m_{k}+3^{k-1}}\right)$.

Note that $F_{k, j}^{(2)}$ is centered but not the two others processes defined above. Their mean functions are denoted by

$$
\Lambda_{k, 1}\left(\mathbf{u}_{k, j}\right):=\mathbb{E} F_{k, j}^{(1)}\left(\mathbf{u}_{k, j}\right) \text { and } \Lambda_{k, 3}\left(\mathbf{u}_{k, j+1}\right)=\mathbb{E} F_{k, j}^{(3)}\left(\mathbf{u}_{k, j+1}\right) .
$$

Note that, for any $j=1, \ldots, q_{k}+1$, we have

$$
B_{k, j}=F_{k, j}^{(1)}\left(\boldsymbol{\eta}_{k, j}\right)+F_{k, j}^{(2)}+F_{k, j}^{(3)}\left(\boldsymbol{\eta}_{k, j+1}\right) .
$$

Let us now introduce the centered process

$$
Y_{k, j}\left(\mathbf{u}_{k, j}, \mathbf{u}_{k, j+1}\right)=F_{k, j}^{(0,1)}\left(\mathbf{u}_{k, j}\right)+F_{k, j}^{(2)}+F_{k, j}^{(0,3)}\left(\mathbf{u}_{k, j+1}\right),
$$

where

$$
F_{k, j}^{(0,1)}\left(\mathbf{u}_{k, j}\right)=F_{k, j}^{(1)}\left(\mathbf{u}_{k, j}\right)-\Lambda_{k, 1}\left(\mathbf{u}_{k, j}\right) \text { and } F_{k, j}^{(0,3)}\left(\mathbf{u}_{k, j+1}\right)=F_{k, j}^{(3)}\left(\mathbf{u}_{k, j+1}\right)-\Lambda_{k, 3}\left(\mathbf{u}_{k, j+1}\right) .
$$

Note that $Y_{k, j}\left(\mathbf{u}_{k, j}, \mathbf{u}_{k, j+1}\right), j=1, \ldots, q_{k}, k \geq K_{0}$ are then mean zero independent random variables with variance function denoted by

$$
V_{k}\left(\mathbf{u}_{k, j}, \mathbf{u}_{k, j+1}\right):=\left\|Y_{k, j}\left(\mathbf{u}_{k, j}, \mathbf{u}_{k, j+1}\right)\right\|_{2}^{2} .
$$


Define then

$$
b_{n}(\mathbf{u})=\sum_{k=K_{0}}^{b_{n}-1} \sum_{j=1}^{q_{k}} Y_{k, j}\left(\mathbf{u}_{k, j}, \mathbf{u}_{k, j+1}\right)+\sum_{j=1}^{\tau_{n}} Y_{b_{n}, j}\left(\mathbf{u}_{b_{n}, j}, \mathbf{u}_{b_{n}, j+1}\right) .
$$

Using Theorem 1 in Sakhanenko [25] as it will be done later, it is possible to infer that we can strongly approximate $b_{n}(\mathbf{u})$ by a Brownian motion and that the error in the strong approximation is of the right order. However, the variance of the approximating Brownian motion will be the variance of $b_{n}(\mathbf{u})$ that is

$$
\operatorname{var}\left(b_{n}(\mathbf{u})\right):=Q_{n}(\mathbf{u})=\sum_{k=K_{0}}^{b_{n}-1} \sum_{j=1}^{q_{k}} V_{k}\left(\mathbf{u}_{k, j}, \mathbf{u}_{k, j+1}\right)+\sum_{j=1}^{\tau_{n}} V_{b_{n}}\left(\mathbf{u}_{b_{n}, j}, \mathbf{u}_{b_{n}, j+1}\right)
$$

Since, for $k$ fixed, the random variables $\left(V_{k}\left(\boldsymbol{\eta}_{k, j}, \boldsymbol{\eta}_{k, j+1}\right)\right)_{j \geq 1}$ are not independent, this creates problems to proceed to the unconditional Gaussian approximation as done in Step 3.3 in BerkesLiu-Wu [3]. This is the reason why Berkes-Liu-Wu 3 , have introduced another process $\Gamma_{n}(\mathbf{u})$ and rather than approximating $b_{n}(\mathbf{u})$, they approximate the process

$$
H_{n}^{\circ}(\mathbf{u}):=b_{n}(\mathbf{u})+\Gamma_{n}(\mathbf{u}) .
$$

The process $\Gamma_{n}(\mathbf{u})$ is defined as follows:

$$
\Gamma_{n}(\mathbf{u})=\sum_{k=K_{0}}^{b_{n}-1} L_{k}^{1 / 2}\left(\mathbf{u}_{k, 1}\right) \zeta_{k}+L_{b_{n}}^{1 / 2}\left(\mathbf{u}_{b_{n}, 1}\right) \zeta_{b_{n}}
$$

where $\left(\zeta_{\ell}\right)_{\ell \in \mathbb{Z}}$ is a sequence of iid standard normal random variables which is independent of $\left(\varepsilon_{\ell}\right)_{\ell \in \mathbb{Z}}$

$$
L_{k}\left(\mathbf{u}_{k, j}\right)=\left\|F_{k, j}^{(2)}+F_{k, j}^{(0,3)}\left(\mathbf{u}_{k, j}\right)\right\|_{2}^{2}, j=1, \ldots, q_{k}+1,
$$

with, for any $j=1, \ldots, q_{k}+1$,

$$
F_{k, j}^{(0,3)}\left(\mathbf{u}_{k, j}\right)=F_{k, j}^{(3)}\left(\mathbf{u}_{k, j}\right)-\Lambda_{k, 3}\left(\mathbf{u}_{k, j}\right),
$$

$$
F_{k, j}^{(3)}\left(\mathbf{u}_{k, j}\right)=\sum_{i=1+(6 j+3) m_{k}}^{(6 j+5) m_{k}} G_{k}\left(\varepsilon_{i+3^{k-1}}, \ldots, \varepsilon_{(6 j+5) m_{k}+3^{k-1}}, u_{(6 j-1) m_{k}+1+3^{k-1}}, \ldots, u_{i-4 m_{k}+3^{k-1}}\right),
$$

and

$$
\Lambda_{k, 3}\left(\mathbf{u}_{k, j}\right)=\mathbb{E} F_{k, j}^{(3)}\left(\mathbf{u}_{k, j}\right) .
$$

Note now that the variance of $H_{n}^{\circ}(\mathbf{u})$ is

$$
Q_{n}^{\circ}(\mathbf{u})=\sum_{k=K_{0}}^{b_{n}-1}\left\{\sum_{j=1}^{q_{k}} V_{k}\left(\mathbf{u}_{k, j}, \mathbf{u}_{k, j+1}\right)+L_{k}\left(\mathbf{u}_{k, 1}\right)\right\}+\sum_{j=1}^{\tau_{n}} V_{b_{n}}\left(\mathbf{u}_{b_{n}, j}, \mathbf{u}_{b_{n}, j+1}\right)+L_{b_{n}}\left(\mathbf{u}_{b_{n}, 1}\right) .
$$

But denoting by

$$
V_{k}^{0}\left(\mathbf{u}_{k, j}\right):=\left\|F_{k, j}^{(0,1)}\left(\mathbf{u}_{k, j}\right)+F_{k, j}^{(2)}+F_{k, j}^{(0,3)}\left(\mathbf{u}_{k, j}\right)\right\|_{2}^{2},
$$

the following equality holds: for any positive integer $t$,

$$
L_{k}\left(\mathbf{u}_{k, 1}\right)+\sum_{j=1}^{t} V_{k}\left(\mathbf{u}_{k, j}, \mathbf{u}_{k, j+1}\right)=\sum_{j=1}^{t} V_{k}^{0}\left(\mathbf{u}_{k, j}\right)+L_{k}\left(\mathbf{u}_{k, t+1}\right) .
$$


Therefore, the variance of $H_{n}^{\circ}(\mathbf{u})$ can be rewritten as:

$$
Q_{n}^{\circ}(\mathbf{u})=\sum_{k=K_{0}}^{b_{n}-1}\left\{\sum_{j=1}^{q_{k}} V_{k}^{0}\left(\mathbf{u}_{k, j}\right)+L_{k}\left(\mathbf{u}_{k, q_{k}+1}\right)\right\}+\sum_{j=1}^{\tau_{n}}\left\{V_{b_{n}}^{0}\left(\mathbf{u}_{b_{n}, j}\right)+L_{b_{n}}\left(\mathbf{u}_{b_{n}, \tau_{n}+1}\right)\right\} .
$$

Since, the random variables $V_{k}^{0}\left(\boldsymbol{\eta}_{k, j}\right), j=1, \ldots, q_{k}, k \geq K_{0}$, are independent, it will be then possible to proceed to an unconditional Gaussian approximation (see our step 4). As in BerkesLiu-Wu [3], for notational convenience in what follows, for $j=0$, we let $Y_{k, 0}\left(\mathbf{u}_{k, 0}, \mathbf{u}_{k, 1}\right):=$ $L_{k}^{1 / 2}\left(\mathbf{u}_{k, 1}\right) \zeta_{k}$. With all the notations above, it follows that

$$
H_{n}^{\circ}(\mathbf{u}):=b_{n}(\mathbf{u})+\Gamma_{n}(\mathbf{u})=\sum_{k=K_{0}}^{b_{n}-1} \sum_{j=0}^{q_{k}} Y_{k, j}\left(\mathbf{u}_{k, j}, \mathbf{u}_{k, j+1}\right)+\sum_{j=0}^{\tau_{n}} Y_{b_{n}, j}\left(\mathbf{u}_{b_{n}, j}, \mathbf{u}_{b_{n}, j+1}\right) .
$$

The step 3.2 in Berkes-Liu-Wu 3 ] consists in applying Theorem 1 in Sakhanenko [26]. We shall rather use Theorem 1 in Sakhanenko [25] (for an easy reference see Theorem A in [27]). With this aim, we set, for any $k \geq K_{0}$ and any $j \geq 0$,

$$
\zeta_{k, j}=k^{-1 / \gamma} Y_{k, j}\left(\mathbf{u}_{k, j}, \mathbf{u}_{k, j+1}\right) .
$$

Note now that for any $k \geq K_{0}$ and any $j \geq 1$,

$$
\left\|Y_{k, j}\left(\mathbf{u}_{k, j}, \mathbf{u}_{k, j+1}\right)\right\|_{\infty} \leq 10|\psi|_{\infty} m_{k} \leq \kappa_{1} k^{1 / \gamma}
$$

(where $\kappa_{1}=10 \kappa|\psi|_{\infty}$ ) implying that, for any $t>0$,

$$
t \mathbb{E}\left(\left|\zeta_{k, j}\right|^{3} \mathrm{e}^{t\left|\zeta_{k, j}\right|}\right) \leq t \kappa_{1} \mathrm{e}^{t \kappa_{1}} \mathbb{E}\left(\left|\zeta_{k, j}\right|^{2}\right) .
$$

So, if $0<t \leq 1 /\left(2 \kappa_{1}\right)$ (implying $\left.t \kappa_{1} \mathrm{e}^{t \kappa_{1}} \leq 1\right)$, it follows that, for any $k \geq K_{0}$ and any $j \geq 1$,

$$
t \mathbb{E}\left(\left|\zeta_{k, j}\right|^{3} \mathrm{e}^{t\left|\zeta_{k, j}\right|}\right) \leq \mathbb{E}\left(\left|\zeta_{k, j}\right|^{2}\right) .
$$

On another hand

$$
\left|L_{k}^{1 / 2}\left(\mathbf{u}_{k, 1}\right)\right| \leq 6|\psi|_{\infty} m_{k} \leq \kappa_{1} k^{1 / \gamma} .
$$

Moreover, for any positive integer $\sigma$, any positive $t$ and any standard Gaussian r.v. $Z$,

$$
\mathbb{E}\left(|Z|^{3} \mathrm{e}^{t \sigma|Z|}\right) \leq 2 \mathrm{e}^{t^{2} \sigma^{2} / 2}\left(\frac{\left(2+(\sigma t)^{2}\right) \mathrm{e}^{-t^{2} \sigma^{2} / 2}}{\sqrt{2 \pi}}+3 \sigma t+(\sigma t)^{3}\right):=g(\sigma t) .
$$

Applying the inequality above with $\sigma=\kappa_{1}$, it follows that, for any $k \geq K_{0}$ and any $t>0$,

$$
t \mathbb{E}\left(\left|\zeta_{k, 0}\right|^{3} \mathrm{e}^{t\left|\zeta_{k, 0}\right|}\right) \leq\left|k^{-1 / \gamma} L_{k}^{1 / 2}\left(\mathbf{u}_{k, 1}\right)\right|^{2}\left(\kappa_{1} t\right) g\left(\kappa_{1} t\right):=\mathbb{E}\left(\left|\zeta_{k, 0}\right|^{2}\right)\left(\kappa_{1} t\right) g\left(\kappa_{1} t\right) .
$$

Since there exists $\kappa_{2}$ such that for any positive $t$ such that $t \leq 1 / \kappa_{2}$, we have $\left(\kappa_{1} t\right) g\left(\kappa_{1} t\right) \leq 1$, we get that for any $t \leq 1 / \kappa_{2}$ and any $k \geq K_{0}$,

$$
t \mathbb{E}\left(\left|\zeta_{k, 0}\right|^{3} \mathrm{e}^{t\left|\zeta_{k, 0}\right|}\right) \leq \mathbb{E}\left(\left|\zeta_{k, 0}\right|^{2}\right) .
$$

So, overall, for $t:=K=\left(\max \left(2 \kappa_{1}, \kappa_{2}\right)\right)^{-1}$, we get that, for any $k \geq K_{0}$ and any $j \geq 0$,

$$
t \mathbb{E}\left(\left|\zeta_{k, j}\right|^{3} \mathrm{e}^{t\left|\zeta_{k, j}\right|}\right) \leq \mathbb{E}\left(\left|\zeta_{k, j}\right|^{2}\right) .
$$


Using Theorem 1 in Sakhanenko [25], it follows that there exists a probability space $\left(\Omega_{\mathbf{u}}, \mathcal{A}_{\mathbf{u}}, \mathbb{P}_{\mathbf{u}}\right)$ on which we can define random variables $R_{k, j}^{\mathbf{u}}$ such that

$$
\left(R_{k, j}^{\mathbf{u}}\right)_{0 \leq j \leq q_{k}, k \geq K_{0}}=^{\mathcal{D}}\left(Y_{k, j}\left(\mathbf{u}_{k, j}, \mathbf{u}_{k, j+1}\right)\right)_{0 \leq j \leq q_{k}, k \geq K_{0}},
$$

and a sequence of independent normal random variables $\left(N_{k, j}^{\mathbf{u}}\right)_{0 \leq j \leq q_{k}, k \geq K_{0}}$ with mean zero and $\operatorname{Var}\left(N_{k, j}^{\mathbf{u}}\right)=k^{-2 / \gamma} \operatorname{Var}\left(Y_{k, j}\left(\mathbf{u}_{k, j}, \mathbf{u}_{k, j+1}\right)\right)$ in such a way that, for any positive integer $\ell$ and any positive real $x$,

$$
\begin{gathered}
\mathbb{P}_{\mathbf{u}}\left(\max _{N_{0} \leq i \leq 3^{\ell}}\left|\sum_{k=K_{0}}^{h_{i}-1} \sum_{j=0}^{q_{k}} R_{k, j}^{\mathbf{u}}+\sum_{j=0}^{\tau_{i}} R_{h_{i}, j}^{\mathbf{u}}-\sum_{k=K_{0}}^{h_{i}-1} \sum_{j=0}^{q_{k}} k^{1 / \gamma} N_{k, j}^{\mathbf{u}}-\sum_{j=0}^{\tau_{i}} h_{i}^{1 / \gamma} N_{h_{i}, j}^{\mathbf{u}}\right| \geq x\right) \\
\leq \mathbb{P}_{\mathbf{u}}\left(2 \ell^{1 / \gamma} \max _{N_{0} \leq i \leq 3^{\ell}}\left|\sum_{k=K_{0}}^{h_{i}-1} \sum_{j=0}^{q_{k}} k^{-1 / \gamma} R_{k, j}^{\mathbf{u}}+\sum_{j=0}^{\tau_{i}} h_{i}^{-1 / \gamma} R_{h_{i}, j}^{\mathbf{u}}-\sum_{k=K_{0}}^{h_{i}-1} \sum_{j=0}^{q_{k}} N_{k, j}^{\mathbf{u}}-\sum_{j=0}^{\tau_{i}} N_{h_{i}, j}^{\mathbf{u}}\right| \geq x\right) \\
\leq\left(1+K \sum_{k=K_{0}}^{\ell} \sum_{j=0}^{q_{k}} k^{-2 / \gamma} \mathbb{E}\left(Y_{k, j}^{2}\left(\mathbf{u}_{k, j}, \mathbf{u}_{k, j+1}\right)\right) \exp \left(-A K x \ell^{-1 / \gamma} / 2\right),\right.
\end{gathered}
$$

where $A$ is a universal constant and we recall that $K=\left(\max \left(2 \kappa_{1}, \kappa_{2}\right)\right)^{-1}$. Note that the first inequality in the inequations above follows from an application of Lemma 2.1 in Shao [27] which states that if $\left\{u_{n}, n \geq 1\right\}$ is a non-decreasing sequence of positive numbers and if $\left\{\zeta_{n}, n \geq 1\right\}$ is a sequence of random variables, then for each $n \geq 1$,

$$
\left|\sum_{i=1}^{n} u_{i} \zeta_{i}\right| \leq 2 u_{n} \max _{i \leq n}\left|\sum_{j=1}^{i} \zeta_{j}\right|
$$

Note now that

$$
\sum_{k=K_{0}}^{h_{i}-1} \sum_{j=0}^{q_{k}} k^{2 / \gamma} \operatorname{Var}\left(N_{k, j}^{\mathbf{u}}\right)+\sum_{j=0}^{\tau_{i}} h_{i}^{2 / \gamma} \operatorname{Var}\left(N_{h_{i}, j}^{\mathbf{u}}\right)=Q_{i}^{\circ}(\mathbf{u}),
$$

where $Q_{i}^{\circ}(\mathbf{u})$ is defined in (3.14). Hence it follows that there is a Brownian motion $\mathbb{B}_{\mathbf{u}}$ such that for any positive integer $\ell$ and any positive real $x$,

$$
\begin{aligned}
\mathbb{P}_{\mathbf{u}}\left(\max _{N_{0} \leq i \leq 3^{\ell}}\left|\sum_{k=K_{0}}^{h_{i}-1} \sum_{j=0}^{q_{k}} R_{k, j}^{\mathbf{u}}+\sum_{j=0}^{\tau_{i}} R_{h_{i}, j}^{\mathbf{u}}-\mathbb{B}_{\mathbf{u}}\left(Q_{i}^{0}(\mathbf{u})\right)\right| \geq x\right) \\
\leq\left(1+K \Psi_{\ell}(\mathbf{u})\right) \exp \left(-A K x \ell^{-1 / \gamma} / 2\right)
\end{aligned}
$$

where

Note that

$$
\Psi_{\ell}(\mathbf{u})=\sum_{k=K_{0}}^{\ell} \sum_{j=0}^{q_{k}} k^{-2 / \gamma} \mathbb{E}\left(Y_{k, j}^{2}\left(\mathbf{u}_{k, j}, \mathbf{u}_{k, j+1}\right)\right) .
$$

$$
\mathbb{E}\left(\Psi_{\ell}(\boldsymbol{\eta})\right) \ll \sum_{k=K_{0}}^{\ell} k^{-2 / \gamma} q_{k}\left\|\widetilde{W}_{k, m_{k}}\right\|_{2}^{2} \ll 3^{\ell} .
$$

By taking $x=C \ell^{1+1 / \gamma}$ in (3.18) with $C=(4 \log 3) /(A K)$, we conclude via the Borel-Cantelli lemma, that as $n \rightarrow \infty$,

$$
\max _{i \leq n}\left|\sum_{k=K_{0}}^{h_{i}-1} \sum_{j=0}^{q_{k}} R_{k, j}^{\eta}+\sum_{j=0}^{\tau_{i}} R_{h_{i}, j}^{\eta}-\mathbb{B}_{\boldsymbol{\eta}}\left(Q_{i}^{\circ}(\boldsymbol{\eta})\right)\right|=O\left((\log n)^{\alpha}\right) \text { a.s. }
$$

This ends the step 3 of our proof. 


\subsection{Step 4. (Unconditional Gaussian approximation)}

Starting from the conditional Gaussian approximation (3.19), we shall prove here that there exists a Brownian motion $\mathbb{B}$ such that

$$
\max _{1 \leq i \leq n}\left|S_{i}^{\diamond}-\mathbb{B}\left(\sigma_{i}^{2}\right)\right|=O\left((\log n)^{\alpha}\right) \text { a.s. }
$$

where

$$
\sigma_{n}^{2}:=\sum_{k=K_{0}}^{b_{n}-1} q_{k}\left\|A_{k, 1}\right\|_{2}^{2}+\tau_{n}\left\|A_{b_{n}, 1}\right\|_{2}^{2} .
$$

With this aim, we shall use arguments developed in the step 3.3 in Berkes-Liu-Wu [3] with some modifications. This step consists first in showing that we can decompose the Brownian motion $\mathbb{B}_{\mathbf{u}}$, constructed at Step 3, as

$$
\mathbb{B}_{\mathbf{u}}\left(Q_{n}^{\circ}(\mathbf{u})\right)=\bar{w}_{n}(\mathbf{u})+\varphi_{n}(\mathbf{u}),
$$

where

$$
\max _{i \leq n}\left|\varphi_{i}(\boldsymbol{\eta})\right|=O\left((\log n)^{\alpha}\right) \text { a.s. }
$$

and that

$$
\left(\Phi_{i}\right)_{i \geq N_{0}}{ }^{\mathcal{D}}\left(\bar{w}_{i}(\boldsymbol{\eta})\right)_{i \geq N_{0}}
$$

where

$$
\Phi_{n}=\sum_{k=K_{0}}^{b_{n}-1} \sum_{j=1}^{q_{k}}\left(V_{k}^{0}\left(\boldsymbol{\eta}_{k, j}\right)\right)^{1 / 2} Z_{k, j}^{\star}+\sum_{j=1}^{\tau_{n}}\left(V_{b_{n}}^{0}\left(\boldsymbol{\eta}_{b_{n}, j}\right)\right)^{1 / 2} Z_{b_{n}, j}^{\star},
$$

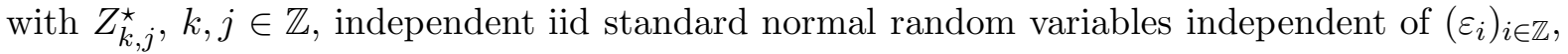
and

$$
\varphi_{n}(\mathbf{u})=\sum_{k=K_{0}}^{b_{n}-1} L_{k}^{1 / 2}\left(\mathbf{u}_{k, q_{k}+1}\right) \mathcal{G}_{k, 1+q_{k}}^{\mathbf{u}}+L_{b_{n}}^{1 / 2}\left(\mathbf{u}_{b_{n}, \tau_{n}+1}\right) \mathcal{G}_{b_{n}, 1+\tau_{n}}^{\mathbf{u}},
$$

where $\left(\mathcal{G}_{k, j}^{\mathbf{u}}\right)_{k, j}$ are standard normal random variables (that can be possibly dependent) but which are independent of $\boldsymbol{\eta}$.

Note that $\tau_{n} \leq q_{b_{n}}$. Hence to prove that $\left|L_{b_{n}}^{1 / 2}\left(\boldsymbol{\eta}_{b_{n}, \tau_{n}+1}\right) \mathcal{G}_{b_{n}, 1+\tau_{n}}^{\eta}\right|=O\left((\log n)^{\alpha}\right)$ a.s., as $n \rightarrow \infty$ (where we recall that $\alpha=1+\frac{1}{\gamma}$ ), it is enough to show that

$$
\sum_{k \geq K_{0}} \mathbb{P}\left(\max _{1 \leq j \leq q_{k}}\left|L_{k}^{1 / 2}\left(\boldsymbol{\eta}_{k, j+1}\right) \mathcal{G}_{k, 1+j}^{\eta}\right|>C k^{1+1 / \gamma}\right)<\infty
$$

Using Markov inequality and the independence between $\left(\mathcal{G}_{k, j}^{\mathbf{u}}\right)_{k, j}$ and $\boldsymbol{\eta}$, the fact that

$$
L_{k}\left(\mathbf{u}_{k, j+1}\right) \leq\left(6 m_{k}|\psi|_{\infty}\right)^{2} \leq \kappa_{3} k^{2 / \gamma},
$$

and that for $N \sim \mathcal{N}(0,1)$, for any $x>0$,

$$
\mathbb{P}(|N| \geq x) \leq \frac{\sqrt{2}}{x \sqrt{\pi}} \exp \left(-x^{2} / 2\right)
$$

we infer that

$$
\mathbb{P}\left(\max _{1 \leq j \leq q_{k}}\left|L_{k}^{1 / 2}\left(\boldsymbol{\eta}_{k, j+1}\right) \mathcal{G}_{k, 1+j}^{\eta}\right|>C k^{1+1 / \gamma}\right) \ll k^{-1} q_{k} \exp \left(-\kappa_{4} k^{2}\right),
$$


where $\kappa_{4}$ is a positive constant depending on $\kappa_{3}$ and $C$. This proves (3.26). To end the proof of (3.23), it remains to prove that $\sum_{k=K_{0}}^{b_{n}-1} L_{k}^{1 / 2}\left(\boldsymbol{\eta}_{k, q_{k}+1}\right) \mathcal{G}_{k, 1+q_{k}}^{\eta}=O\left((\log n)^{\alpha}\right)$ a.s. as $n \rightarrow \infty$. By Kronecker lemma, this holds if

$$
\sum_{k \geq K_{0}} k^{-\alpha} \mathbb{E}\left(L_{k}^{1 / 2}\left(\boldsymbol{\eta}_{k, q_{k}+1}\right)\left|\mathcal{G}_{k, 1+q_{k}}^{\boldsymbol{\eta}}\right|\right)<\infty
$$

But

$$
\mathbb{E}\left(L_{k}^{1 / 2}\left(\boldsymbol{\eta}_{k, q_{k}+1}\right)\left|\mathcal{G}_{k, 1+q_{k}}^{\boldsymbol{\eta}}\right|\right)=\int \mathbb{E}\left(L_{k}^{1 / 2}\left(\boldsymbol{\eta}_{k, q_{k}+1}\right) \mid \mathcal{G}_{k, 1+q_{k}}^{\boldsymbol{\eta}} \| \boldsymbol{\eta}=\mathbf{u}\right) d P_{\boldsymbol{\eta}}(\mathbf{u}) .
$$

Hence, using the independence between $\mathcal{G}_{k, 1+q_{k}}^{\boldsymbol{\eta}}$ and $\boldsymbol{\eta}$ and the fact that $\mathbb{E}\left|\mathcal{G}_{k, 1+q_{k}}^{\mathbf{u}}\right| \leq 1$, it follows that

$$
\mathbb{E}\left(L_{k}^{1 / 2}\left(\boldsymbol{\eta}_{k, q_{k}+1}\right)\left|\mathcal{G}_{k, 1+q_{k}}^{\eta}\right|\right) \leq \mathbb{E}\left(L_{k}^{1 / 2}\left(\left(\boldsymbol{\eta}_{k, q_{k}+1}\right)\right) .\right.
$$

Using the fact that the $\varepsilon_{i}$ 's are iid, we infer that

$$
\mathbb{E}\left(L_{k}^{1 / 2}\left(\boldsymbol{\eta}_{k, q_{k}+1}\right)\right) \leq 2\left\|F_{k, q_{k}+1}^{(2)}\right\|_{2} .
$$

But, by stationarity and the estimate (4.10) in [7],

$$
\left\|F_{k, q_{k}+1}^{(2)}\right\|_{2} \ll\left\|\sum_{i=1}^{m_{k}} X_{i}\right\|_{2}+m_{k}\left(2|\psi|_{\infty} \mathbb{P}\left(T \geq m_{k}\right)\right)^{1 / 2}+\sum_{i=1}^{m_{k}}\left\|X_{i}-X_{k, i}\right\|_{2} .
$$

Hence, by Lemma 2.1 and Proposition 2.3 ,

$$
\mathbb{E}\left(L_{k}^{1 / 2}\left(\boldsymbol{\eta}_{k, q_{k}+1}\right)\right) \ll\left\|\sum_{i=1}^{m_{k}} X_{i}\right\|_{2}+k^{1 / \gamma} \exp \left(-\delta\left(2^{-1} \kappa\right)^{\gamma} k\right) .
$$

Since by Lemma 2.2. $\sum_{i \geq 0}\left|\operatorname{Cov}\left(X_{0}, X_{i}\right)\right|<\infty$, it follows that

$$
\mathbb{E}\left(L_{k}^{1 / 2}\left(\boldsymbol{\eta}_{k, q_{k}+1}\right)\right) \ll \sqrt{m_{k}} .
$$

Hence

$$
\sum_{k \geq K_{0}} k^{-\alpha} \mathbb{E}\left(L_{k}^{1 / 2}\left(\boldsymbol{\eta}_{k, q_{k}+1}\right)\left|\mathcal{G}_{k, 1+q_{k}}^{\eta}\right|\right) \ll \sum_{k \geq K_{0}} k^{-\alpha} k^{1 /(2 \gamma)}<\infty,
$$

since $\alpha-1 /(2 \gamma)=1+1 /(2 \gamma)>1$. This ends the proof of (3.23) .

Now, the same arguments as to prove (3.23) show that

$$
\max _{i \leq n}\left|\Gamma_{i}(\boldsymbol{\eta})\right|=O\left((\log n)^{\alpha}\right) \text { and then } \max _{i \leq n}\left|\sum_{k=K_{0}}^{h_{i}-1} R_{k, 0}^{\eta}+R_{h_{i}, 0}^{\eta}\right|=O\left((\log n)^{\alpha}\right) \text { a.s. }
$$

where we recall that $\Gamma_{n}(\mathbf{u})$ has been defined in (3.13). So, overall, taking into account (3.19), (3.22), (3.23) and (3.27), we get

$$
\max _{i \leq n}\left|\sum_{k=K_{0}}^{h_{i}-1} \sum_{j=1}^{q_{k}} R_{k, j}^{\eta}+\sum_{j=1}^{\tau_{i}} R_{h_{i}, j}^{\eta}-\bar{\omega}_{i}(\boldsymbol{\eta})\right|=O\left((\log n)^{\alpha}\right) \text { a.s. }
$$

But note now that

$$
\left(\sum_{k=K_{0}}^{h_{i}-1} \sum_{j=1}^{q_{k}} R_{k, j}^{\eta}+\sum_{j=1}^{\tau_{i}} R_{h_{i}, j}^{\eta}+M_{i}(\boldsymbol{\eta})\right)_{i \geq N_{0}}={ }^{\mathcal{D}}\left(S_{i}^{\diamond}\right)_{i \geq N_{0}}
$$


where

$$
M_{n}(\mathbf{u})=\sum_{k=K_{0}}^{b_{n}-1} \sum_{j=1}^{q_{k}}\left\{\Lambda_{k, 1}\left(\mathbf{u}_{k, j}\right)+\Lambda_{k, 3}\left(\mathbf{u}_{k, j+1}\right)\right\}+\sum_{j=1}^{\tau_{n}}\left\{\Lambda_{b_{n}, 1}\left(\mathbf{u}_{k, j}\right)+\Lambda_{b_{n}, 3}\left(\mathbf{u}_{b_{n}, j+1}\right)\right\} .
$$

Recalling (3.24), it remains to prove a strong invariance principle for $\Phi_{n}+M_{n}(\boldsymbol{\eta})$ (where $\Phi_{n}$ is defined in (3.25) ). With this aim, let

$$
A_{k, j}=\left(V_{k}^{\circ}\left(\boldsymbol{\eta}_{k, j}\right)\right)^{1 / 2} Z_{k, j}^{\star}+\Lambda_{k, 1}\left(\boldsymbol{\eta}_{k, j}\right)+\Lambda_{k, 3}\left(\boldsymbol{\eta}_{k, j}\right),
$$

where we recall that $\Lambda_{k, 1}\left(\mathbf{u}_{k, j}\right)=\mathbb{E} F_{k, j}^{(1)}\left(\mathbf{u}_{k, j}\right)$ and $\Lambda_{k, 3}\left(\mathbf{u}_{k, j}\right)=\mathbb{E} F_{k, j}^{(3)}\left(\mathbf{u}_{k, j}\right)$. Note that the random variables $A_{k, j}, j=1, \ldots q_{k}, k \geq K_{0}$ are independent. Denote by

$$
S_{n}^{\sharp}:=\sum_{k=K_{0}}^{b_{n}-1} \sum_{j=1}^{q_{k}} A_{k, j}+\sum_{j=1}^{\tau_{n}} A_{b_{n}, j}
$$

and

$R_{n}^{\sharp}:=\Phi_{n}+M_{n}(\boldsymbol{\eta})-S_{n}^{\sharp}=\sum_{k=K_{0}}^{b_{n}-1}\left\{\Lambda_{k, 3}\left(\boldsymbol{\eta}_{k, q_{k}+1}\right)-\Lambda_{k, 3}\left(\boldsymbol{\eta}_{k, 1}\right)\right\}+\left\{\Lambda_{b_{n}, 3}\left(\boldsymbol{\eta}_{b_{n}, \tau_{n}+1}\right)-\Lambda_{b_{n}, 3}\left(\boldsymbol{\eta}_{b_{n}, 1}\right)\right\}$.

For any $j \geq 0$, note that

$$
\left|\Lambda_{k, 3}\left(\boldsymbol{\eta}_{k, j+1}\right)\right| \leq 2 m_{k}|\psi|_{\infty} \ll k^{1 / \gamma} \text { a.s. }
$$

Therefore

$$
\max _{1 \leq i \leq n}\left|R_{i}^{\sharp}\right|=O\left((\log n)^{1+1 / \gamma}\right) \text { a.s. }
$$

Hence to prove the strong invariance principle for $\Phi_{n}+M_{n}(\boldsymbol{\eta})$ (and then for $S_{n}^{\diamond}$ ) with rate $O\left((\log n)^{\alpha}\right)$, it suffices to prove a strong invariance principle for $S_{n}^{\sharp}$ with the same rate. With this aim, recall that the random variables $A_{k, j}, j=1, \ldots q_{k}, k \geq K_{0}$ are independent. We shall then use again Theorem 1 in Sakhanenko [25]. Note first that there exists a positive constant $\kappa_{5}$ depending only on $\delta, \gamma$ and $|\psi|_{\infty}$ such that

$$
\left(V_{k}^{\circ}\left(\boldsymbol{\eta}_{k, j}\right)\right)^{1 / 2} \leq \kappa_{5} k^{1 / \gamma} \text { a.s. and } \Lambda_{k, 0}\left(\boldsymbol{\eta}_{k, j}\right)+\Lambda_{k, 2}\left(\boldsymbol{\eta}_{k, j}\right) \leq \kappa_{5} k^{1 / \gamma} \text { a.s. }
$$

Hence, if we define for any integers $k \geq K_{0}$ and $j \geq 1$,

$$
\xi_{k, j}=k^{-1 / \gamma} A_{k, j}
$$

we get, for any $t>0$,

$$
\begin{aligned}
\mathbb{E}\left(\left|\xi_{k, j}\right|^{3} \mathrm{e}^{t\left|\xi_{k, j}\right|}\right) & \\
& \leq 4 k^{-3 / \gamma} \mathbb{E}\left(\left(V_{k}^{\circ}\left(\boldsymbol{\eta}_{k, j}\right)\right)^{3 / 2}|N|^{3} \mathrm{e}^{t\left|\xi_{k, j}\right|}\right)+4 k^{-3 / \gamma} \mathbb{E}\left(\left|\Lambda_{k, 0}\left(\boldsymbol{\eta}_{k, j}\right)+\Lambda_{k, 2}\left(\boldsymbol{\eta}_{k, j}\right)\right|^{3} \mathrm{e}^{t\left|\xi_{k, j}\right|}\right) \\
& \leq 4 \kappa_{5} k^{-2 / \gamma} \mathrm{e}^{t \kappa_{5}} \mathbb{E}\left(\left(V_{k}^{\circ}\left(\boldsymbol{\eta}_{k, j}\right)\right)|N|^{3} \mathrm{e}^{t \kappa_{5}|N|}\right)+4 \kappa_{5} k^{-2 / \gamma} \mathrm{e}^{t \kappa_{5}} \mathbb{E}\left(\left|\Lambda_{k, 0}\left(\boldsymbol{\eta}_{k, j}\right)+\Lambda_{k, 2}\left(\boldsymbol{\eta}_{k, j}\right)\right|^{2} \mathrm{e}^{t \kappa_{5}|N|}\right),
\end{aligned}
$$

where $N$ is a standard Gaussian real-valued r.v. independent of $\left(\varepsilon_{i}\right)_{i \in \mathbb{Z}}$. Therefore, it follows that for any $t>0$ and any integers $k \geq K_{0}$ and $j \geq 1$,

$$
\begin{aligned}
& \mathbb{E}\left(\left|\xi_{k, j}\right|^{3} \mathrm{e}^{t\left|\xi_{k, j}\right|}\right) \\
\leq & 4 \kappa_{5} k^{-2 / \gamma} \mathrm{e}^{t \kappa_{5}} \mathbb{E}\left(V_{k}^{\circ}\left(\boldsymbol{\eta}_{k, j}\right)\right) \mathbb{E}\left(|N|^{3} \mathrm{e}^{t \kappa_{5}|N|}\right)+4 \kappa_{5} k^{-2 / \gamma} \mathrm{e}^{t \kappa_{5}} \mathbb{E}\left(\left|\Lambda_{k, 0}\left(\boldsymbol{\eta}_{k, j}\right)+\Lambda_{k, 2}\left(\boldsymbol{\eta}_{k, j}\right)\right|^{2}\right) \mathbb{E}\left(\mathrm{e}^{t \kappa_{5}|N|}\right) .
\end{aligned}
$$


Since $Z_{k, j}^{\star}, k, j \in \mathbb{Z}$ are centered with variance 1 and independent of $\left(\varepsilon_{i}\right)_{i \in \mathbb{Z}}$, it follows that

$$
\mathbb{E}\left(A_{k, j}^{2}\right)=\mathbb{E}\left(V_{k}^{\circ}\left(\boldsymbol{\eta}_{k, j}\right)\right)+\mathbb{E}\left(\left|\Lambda_{k, 1}\left(\boldsymbol{\eta}_{k, j}\right)+\Lambda_{k, 3}\left(\boldsymbol{\eta}_{k, j}\right)\right|^{2}\right)
$$

Therefore, for any $t>0$ and any integers $k, j$,

$$
t \mathbb{E}\left(\left|\xi_{k, j}\right|^{3} \mathrm{e}^{t\left|\xi_{k, j}\right|}\right) \leq 4 t \kappa_{5} \mathrm{e}^{t \kappa_{5}} \mathbb{E}\left(\xi_{k, j}^{2}\right) \mathbb{E}\left(\left(|N|^{3} \vee 1\right) \mathrm{e}^{t \kappa_{5}|N|}\right) .
$$

Hence, taking into account (3.15) $)$ and the fact that $\mathbb{E}\left(\mathrm{e}^{t \kappa_{5}|N|}\right) \leq 2 \mathrm{e}^{t^{2} \kappa_{5}^{2} / 2}$, it follows that there exists a positive constant $\kappa_{6}$ depending only on $\kappa_{5}$ such that for $t=\kappa_{6}$,

$$
t \mathbb{E}\left(\left|\xi_{k, j}\right|^{3} \mathrm{e}^{t\left|\xi_{k, j}\right|}\right) \leq \mathbb{E}\left(\xi_{k, j}^{2}\right) .
$$

Using Theorem 1 in Sakhanenko [25] and (3.17), we then infer that there exists a Brownian motion $\mathbb{B}$ such that

$$
\max _{1 \leq i \leq n}\left|S_{i}^{\sharp}-\mathbb{B}\left(\sigma_{i}^{2}\right)\right|=O\left((\log n)^{\alpha}\right) \text { a.s. }
$$

where $\sigma_{n}^{2}$ is defined by (3.21). This ends the proof of (3.20) and then of Step 4 .

\subsection{Step 5. (Identifying the variance in the Brownian motion)}

The aim of this step is to show that it is possible to replace in (3.20) the variance function $\sigma_{i}^{2}$ by $i c^{2}$. A careful analysis of Step 3.4 in [3] reveals that this holds provided that setting $\nu_{k}:=\left\|A_{k, 1}\right\|_{2}^{2} /\left(6 m_{k}\right)$,

$$
(\log n) \max _{\ell \leq b_{n}}\left(m_{\ell} \nu_{\ell}\right)^{1 / 2}=O\left((\log n)^{\alpha}\right)
$$

and

$$
3^{\ell}\left(\nu_{\ell}^{1 / 2}-c\right)^{2}=O\left(\ell^{2 \alpha}(\log \ell)^{-1}\right) .
$$

Note also that since $c^{2}$ is assumed to be positive, to prove (3.32), it suffices to prove that

$$
3^{\ell}\left(\nu_{\ell}-c^{2}\right)^{2}=O\left(\ell^{2 \alpha}(\log \ell)^{-1}\right), \text { as } \ell \rightarrow \infty .
$$

Before proving the above convergences, we first notice that by Lemma $2.2, \sum_{i \geq 0}\left|\operatorname{Cov}\left(X_{0}, X_{i}\right)\right|<$ $\infty$. Hence

$$
c^{2}=\lim _{n \rightarrow \infty} \frac{1}{n} \int\left|S_{n}(\varphi)\right|^{2} d \mu=\lim _{n \rightarrow \infty} \frac{1}{n}\left\|S_{n}\right\|_{2}^{2}=\operatorname{Var}\left(X_{0}\right)+2 \sum_{i \geq 0} \operatorname{Cov}\left(X_{0}, X_{i}\right) .
$$

Moreover, proceeding as to get the relation [8, (66)], we have

$$
\nu_{\ell}=\tilde{c}_{\ell, 0}+2 \sum_{k=1}^{2 m_{\ell}} \tilde{c}_{\ell, k},
$$

where, for any $i \geq 0$,

$$
\tilde{c}_{\ell, i}=\operatorname{Cov}\left(\tilde{X}_{\ell, m_{\ell}+1}, \tilde{X}_{\ell, i+m_{\ell}+1}\right) .
$$

Note that, by stationarity, for all $i \geq 0$,

$$
\begin{array}{r}
\left|\tilde{c}_{\ell, i}-\operatorname{Cov}\left(X_{0}, X_{i}\right)\right|=\left|\operatorname{Cov}\left(\tilde{X}_{\ell, m_{\ell}+1}-X_{m_{\ell}+1}, \tilde{X}_{\ell, i+m_{\ell}+1}\right)+\operatorname{Cov}\left(X_{m_{\ell}+1}, \tilde{X}_{\ell, i+m_{\ell}+1}-X_{i+m_{\ell}+1}\right)\right| \\
\leq 2|\psi|_{\infty}\left(\left\|\tilde{X}_{\ell, m_{\ell}+1}-X_{m_{\ell}+1}\right\|_{1}+\left\|\tilde{X}_{\ell, i+m_{\ell}+1}-X_{i+m_{\ell}+1}\right\|_{1}\right) .
\end{array}
$$


But by the estimate (4.10) in [7, Proposition 2.3 and Lemma 2.1, for any $k>m_{\ell}$,

$$
\left\|\tilde{X}_{\ell, k}-X_{k}\right\|_{1} \ll \mathbb{P}\left(T \geq m_{\ell}\right)+\exp \left(-\delta m_{\ell}^{\gamma}\right) \ll \exp \left(-\delta\left(2^{-1} \kappa\right)^{\gamma} \ell\right) .
$$

Moreover, according to Lemma 2.2 ,

$$
\sum_{k>2 m_{\ell}}\left|\operatorname{Cov}\left(X_{0}, X_{k}\right)\right| \ll \exp \left(-\delta m_{\ell}^{\gamma} / 2\right) \ll \exp \left(-\delta\left(2^{-1} \kappa\right)^{\gamma} \ell / 2\right) .
$$

Therefore, setting $\tilde{c}=\delta\left(2^{-1} \kappa\right)^{\gamma} / 2$,

$$
\left|\nu_{\ell}-c^{2}\right| \ll \exp (-\tilde{c} \ell) .
$$

This shows that $\nu_{\ell} \rightarrow c^{2}$, as $\ell \rightarrow \infty$. Hence (3.31) is satisfied since $(\log n) \max _{k \leq b_{n}}\left(m_{k}\right)^{1 / 2} \ll$ $(\log n)^{1+1 /(2 \gamma)}=O\left((\log n)^{\alpha}\right)$. Now, (3.34) proves (3.33) since $2 \tilde{c} \geq \log 3$. The proof of step 5 is complete. This ends the proof of the theorem when $c^{2}>0$.

\section{A Example of nonuniformly expanding system with stretched exponential return times}

Suppose that $f: X \rightarrow X$ is a nonuniformly expanding dynamical system with base $Y \subset X$, reference measure $m$ on $Y$ and return time $\tau: Y \rightarrow \mathbb{N}$. In all standard examples, the return time tails $m(\tau>n)$ decay exponentially, except for Alves-Viana maps with $m(\tau>n)=O\left(\mathrm{e}^{-c \sqrt{n}}\right)$ and intermittent maps [18] with polynomial decay.

Here we present a family of interval maps with an optimal bound on the return times $m(\tau>n) \sim \mathrm{e}^{-\kappa n^{\gamma}}$, where $\left.\left.\gamma \in\right] 0,1\right]$ is a parameter and $\kappa=\kappa(\gamma)>0$. For this class of maps, Theorem 1.6 gives all possible subexponential rates in the ASIP.

Fix $\gamma \in] 0,1]$ and consider the following modification of the intermittent maps from [18]. Let $f:[0,1] \rightarrow[0,1]$,

$$
f(x)= \begin{cases}x\left(1+\frac{c}{|\log x|^{\beta}}\right), & x \leq 1 / 2 \\ 2 x-1, & x>1 / 2\end{cases}
$$

with $\beta=\gamma^{-1}-1$ and $c=(\log 2)^{\beta}$ so that $f(1 / 2)=1$.

Let $Y=] 1 / 2,1]$ be a base, $\tau: Y \rightarrow \mathbb{N}, \tau(x)=\inf \left\{k \geq 1: f^{k}(x) \in Y\right\}$ be the first return time and $F: Y \rightarrow Y, F(x)=f^{\tau(x)}(x)$ be the induced map. Let $\alpha$ denote the partition of $Y$ into the intervals where $\tau$ is constant. Let $m$ denote the Lebesgue measure.

In the rest of this section we prove:

Theorem A.1. $f$ is a nonuniformly expanding map with base $Y$, return time $\tau$ and reference measure $m$. That is, there exists $C>0$ such that for every $a \in \alpha$ and all $x, y \in a$,

(a) $F: a \rightarrow Y$ is a nonsingular bijection;

(b) $F$ is expanding: $|F(y)-F(x)| \geq 2|y-x|$;

(c) F has bounded distortion: $\left|\log F^{\prime}(y)-\log F^{\prime}(x)\right| \leq C|F(y)-F(x)|$.

Further, there exist $\eta_{1}, \eta_{2}>0$ such that for all $n \geq 1$,

(d) $\mathrm{e}^{-\eta_{2} n^{\gamma}} \leq m(\tau \geq n) \leq \mathrm{e}^{-\eta_{1} n^{\gamma}}$.

Proof of Theorem A.1 takes the rest of this section. Items (a) and (b) are straightforward. For (c) and (d), we use the following technical lemma.

Let $g=f_{] 0,1 / 2]}^{-1}$ be the inverse left branch of $f$. For $n \geq 0$, let $z_{n}=g^{n}$ and $u_{n}=-\log z_{n}$. 
Lemma A.2. There exist $\delta_{1}>\delta_{2}>0$ such that for every $n \geq 1$,

$$
\delta_{2} n^{\gamma} \leq u_{n}(x) \leq \delta_{1} n^{\gamma} \quad \forall x \in(1 / 2,1] .
$$

Further, there exists $C>0$ such that for every $n \geq 1$,

$$
\left|\log z_{n}^{\prime}(x)-\log z_{n}^{\prime}(y)\right| \leq C|x-y| \quad \forall x, y \in(1 / 2,1] .
$$

Proof. We have

$$
u_{n}=u_{n+1}-\log \left(1+c / u_{n+1}^{\beta}\right) .
$$

Observe that $\left(x_{n}\right)$ is decreasing to 0 and $\left(u_{n}\right)$ is increasing to $\infty$. Hence,

$$
\begin{aligned}
n+1=\sum_{k=0}^{n} \frac{u_{k+1}-u_{k}}{\log \left(1+c u_{k+1}^{-\beta}\right)} & \geq \sum_{k=0}^{n} \int_{u_{k}}^{u_{k+1}} \frac{d x}{\log \left(1+c x^{-\beta}\right)} \\
& =\int_{u_{0}}^{u_{n+1}} \frac{d x}{\log \left(1+c x^{-\beta}\right)} \geq K u_{n+1}^{\beta+1},
\end{aligned}
$$

for some $K>0$ not depending on $n$. Recall that $\gamma=(1+\beta)^{-1}$. Thus $u_{n} \leq K^{-1} n^{\gamma}$.

By (A.4),$u_{n+1} / u_{n} \rightarrow 1$, hence for some $c^{\prime}, K^{\prime}>0$,

$$
\begin{aligned}
n+1 \leq \sum_{k=0}^{n} \frac{u_{k+1}-u_{k}}{\log \left(1+c^{\prime} u_{k}^{-\beta}\right)} & \leq \sum_{k=0}^{n} \int_{u_{k}}^{u_{k+1}} \frac{d x}{\log \left(1+c^{\prime} x^{-\beta}\right)} \\
& =\int_{u_{0}}^{u_{n+1}} \frac{d x}{\log \left(1+c^{\prime} x^{-\beta}\right)} \leq K^{\prime} u_{n+1}^{\beta+1} .
\end{aligned}
$$

Thus $u_{n} \geq K^{\prime-1} n^{\gamma}$. This completes the proof of (A.2).

It remains to prove (A.3). It suffices to show that

$$
\sup _{n \geq 1} \sup _{x \in(1 / 2,1]}\left|\frac{z_{n}^{\prime \prime}(x)}{z_{n}^{\prime}(x)}\right|<\infty \text {. }
$$

Let $n \geq 1$. We have

$$
\begin{aligned}
& z_{n}=z_{n+1}\left(1+\frac{c}{\left|\log z_{n+1}\right|^{\beta}}\right) \\
& z_{n}^{\prime}=z_{n+1}^{\prime}\left(1+\frac{c}{\left|\log z_{n+1}\right|^{\beta}}+\frac{c \beta}{\left|\log z_{n+1}\right|^{\beta+1}}\right) \\
& z_{n}^{\prime \prime}=z_{n+1}^{\prime \prime}\left(1+\frac{c}{\left|\log z_{n+1}\right|^{\beta}}+\frac{c \beta}{\left|\log z_{n+1}\right|^{\beta+1}}\right)+\frac{\left(z_{n+1}^{\prime}\right)^{2}}{z_{n+1}}\left(\frac{c \beta}{\left|\log z_{n+1}\right|^{\beta+1}}+\frac{c \beta(\beta+1)}{\left|\log z_{n+1}\right|^{\beta+2}}\right) .
\end{aligned}
$$

By the above computations and (A.2), we have

$$
\left|\frac{z_{n}^{\prime}}{z_{n}}\right|=\left|\frac{z_{n+1}^{\prime}}{z_{n+1}}\right|\left(1+\frac{c \beta}{\left|\log z_{n+1}\right|^{\beta+1}\left(1+c\left|\log z_{n+1}\right|^{-\beta}\right)}\right) \geq\left|\frac{z_{n+1}^{\prime}}{z_{n+1}}\right|\left(1+\frac{\varepsilon}{n+1}\right)
$$

for some $\varepsilon>0$. Hence

$$
\left|\frac{z_{n}^{\prime}}{z_{n}}\right| \leq\left|\frac{z_{0}^{\prime}}{z_{0}}\right| \prod_{k=1}^{n}\left(1+\frac{\varepsilon}{k}\right)^{-1} \leq \frac{L}{n^{\eta}}
$$

for some $L>0$ and $\eta>0$.

Next, there is $K>0$ so that

$$
\left|\frac{z_{n+1}^{\prime \prime}}{z_{n+1}^{\prime}}\right| \leq\left|\frac{z_{n}^{\prime \prime}}{z_{n}^{\prime}}\right|+\frac{K}{n+1}\left|\frac{z_{n+1}^{\prime}}{z_{n+1}}\right| .
$$

Combining (A.5) and (A.6), we obtain (A.3). 
Observe that if $x \in Y$ with $\tau(x)=n$, then $F^{\prime}(x)=2 / z_{n-1}^{\prime}(F(x))$. With (A.3), this proves Theorem A.1 (c). Further, $m(\tau \geq n)=z_{n}(1) / 2=\mathrm{e}^{-u_{n}(1)}$. Given (A.2), this implies Theorem A.1 (d).

The proof of Theorem A.1 is complete.

\section{B Proof of Proposition 2.3}

Recall that to simplify the exposition and notation, we assume that $p=\operatorname{gcd}\{h(w): w \in \mathcal{A}\}=1$, so that the underlying Markov chain $\left(g_{k}\right)_{k \geq 0}$ is aperiodic.

Note first that, as quoted in [7], $\psi$ has the following property: For $a, b \in \Omega$, with $a=$ $\left(g_{0}, \ldots, g_{N}, g_{N+1}, \ldots\right), b=\left(g_{0}, \ldots, g_{N}, g_{N+1}^{\prime}, \ldots\right)$ with $g_{N+1} \neq g_{N+1}^{\prime}$,

$$
|\psi(a)-\psi(b)| \leq C \theta^{\sum_{k=0}^{N} \mathbf{1}_{\left\{g_{k} \in S_{0}\right\}}},
$$

where $C$ is a constant depending on $\lambda$ (the constant appearing in (2.4)), the diameter of $X$ and on the Hölder norm of $\varphi$, and $\theta \in] 0,1[$ depends on $\lambda$ and on the Hölder exponent of $\varphi$. Therefore $\delta_{\ell} \leq C \theta^{s_{\ell}}$, where $s_{\ell}=\#\left\{k \leq \ell: g_{k} \in S_{0}\right\}$. Let now $S_{c}=\{(w, h(w)-1), w \in \mathcal{A}\}$. Note that $S_{c}$ is an atom for the Markov chain $\left(g_{n}, n \geq 0\right)$ in the sense that if the chain enters in $S_{c}$ then it regenerates. Moreover,

$$
s_{\ell}=\sum_{i=0}^{\ell} \mathbf{1}_{\left\{g_{i} \in S_{0}\right\}}= \begin{cases}\sum_{i=0}^{\ell-1} \mathbf{1}_{\left\{g_{i} \in S_{c}\right\}} & \text { if } g_{0} \notin S_{0} \\ 1+\sum_{i=0}^{\ell-1} \mathbf{1}_{\left\{g_{i} \in S_{c}\right\}} & \text { if } g_{0} \in S_{0}\end{cases}
$$

Hence, note that

$$
C^{-1} \mathbb{E}\left(\delta_{\ell}\right) \leq \mathbb{E}\left(\theta^{s_{\ell}}\right) \leq \mathbb{E}\left(\theta^{\sum_{i=0}^{\ell-1} \mathbf{1}_{\left\{g_{i} \in S_{c}\right\}}}\right)=\mathbb{E}\left(\theta^{\sum_{i=1}^{\ell} \mathbf{1}_{\left\{g_{i} \in S_{c}\right\}}}\right) .
$$

Let $R_{0}=\inf \left\{n>0: g_{n} \in S_{c}\right\}$ be the first renewal time and for $i \geq 1$,

$$
R_{i}=\inf \left\{n>R_{i-1}: g_{n} \in S_{c}\right\} \text { and } \tau_{i}=R_{i}-R_{i-1} .
$$

Note that $\left(\tau_{i}\right)_{i \geq 1}$ forms a sequence of iid random variables and their common law is the law of $R_{0}$ when the chain starts from $S_{c}$. Let $\kappa=1 /\left(4 \mathbb{E}\left(\tau_{1}\right)\right)$. We have

$$
\mathbb{E}\left(\theta^{\sum_{i=1}^{\ell} \mathbf{1}_{\left\{g_{i} \in S_{c}\right\}}}\right) \leq \theta^{\kappa \ell}+\mathbb{P}\left(\sum_{i=1}^{\ell} \mathbf{1}_{\left\{g_{i} \in S_{c}\right\}}<\kappa \ell\right) .
$$

Next,

$$
\mathbb{P}\left(\sum_{i=1}^{\ell} \mathbf{1}_{\left\{g_{i} \in S_{c}\right\}}<\kappa \ell\right) \leq \mathbb{P}\left(R_{[\kappa \ell]+1}>\ell\right) .
$$

Note that $R_{[\kappa \ell]+1}=R_{0}+\sum_{i=1}^{[\kappa \ell]+1} \tau_{i}$. Hence

$$
\mathbb{P}\left(R_{[\kappa \ell]+1}>\ell\right) \leq \mathbb{P}\left(R_{0}>\ell / 2\right)+\mathbb{P}\left(\sum_{i=1}^{[\kappa \ell]+1} \tau_{i}>\ell / 2\right) .
$$

But

$$
\mathbb{P}\left(\sum_{i=1}^{[\kappa \ell]+1} \tau_{i}>\ell / 2\right) \leq \mathbb{P}\left(\sum_{i=1}^{[\kappa \ell]+1}\left(\tau_{i}-\mathbb{E}\left(\tau_{i}\right)\right)>\ell / 4-\mathbb{E}\left(\tau_{1}\right)\right) \leq \mathbb{P}\left(\sum_{i=1}^{[\kappa \ell]+1}\left(\tau_{i}-\mathbb{E}\left(\tau_{i}\right)\right)>\ell / 8\right),
$$


if $\ell \geq 8 \mathbb{E}\left(\tau_{1}\right)$. Now, using that $\mathbb{P}\left(\tau_{1} \geq k\right)=\mathbb{P}_{\mathcal{A}}(h \geq k)$ and that if $g_{0}=(w, \ell)$, then $R_{0}=$ $h(w)-\ell-1$, and recalling that since we assume a subexponential moment of order $\gamma$ for the return time, $h$ has also a subexponential moment of the same order, we infer that there exists a positive constant $c$ such that

$$
\mathbb{E}\left(\mathrm{e}^{t \tau_{1}^{\gamma}}\right)<\infty \text { and } \mathbb{E}_{\nu}\left(\mathrm{e}^{t R_{0}^{\gamma}}\right)<\infty \text { for any }|t| \leq c .
$$

According to Bernstein's $\psi_{1}$ inequality (see for instance [29, Lemma 2.2.11] and the subsequent remark) when $\gamma=1$ or to the proof of Corollary 5.1 in Borovkov [4] when $\gamma<1$ (see also inequality (1.4) in [22]), there exists a positive constant $c_{1}$ such that

$$
\mathbb{P}\left(\sum_{i=1}^{[\kappa \ell]+1}\left(\tau_{i}-\mathbb{E}\left(\tau_{i}\right)\right)>\ell / 8\right) \leq \exp \left(-c_{1} \ell^{\gamma}\right) .
$$

So, overall, there exists a positive constant $c_{2}$ such that

$$
\mathbb{P}\left(R_{[\kappa \ell]+1}>\ell\right) \leq \exp \left(-c_{2} \ell^{\gamma}\right) .
$$

The proposition follows by taking into account (B.1) and (B.2).

\section{Acknowledgements}

C.C. acknowledges the hospitality of Warwick University and Université Paris-Est (Marne-laVallée). A.K. was supported in part by a European Advanced Grant StochExtHomog (ERC AdG 320977) at the University of Warwick and an Engineering and Physical Sciences Research Council grant EP/P034489/1 at the University of Exeter, and is grateful to Mark Holland and Ian Melbourne for support during this work.

\section{References}

[1] J. Aaronson, M. Denker, Local limit theorems for partial sums of stationary sequences generated by Gibbs-Markov maps, Stoch. Dyn. 1 (2001), 193-237.

[2] V. Baladi, S. Gouëzel, A note on stretched exponential decay of correlations for the VianaAlves map, arXiv:math/0311189 (2003).

[3] I. Berkes, W. Liu, W. and W.B. Wu, Komlós-Major-Tusnády approximation under dependence, Ann. Probab. 42 (2014), 794-817.

[4] A.A.. Borovkov, Estimates for the distribution of sums and maxima of sums of random variables when the Cramér condition is not satisfied. Siberian Math. J. 41 (2000), no. 5, 811-848.

[5] H. Bruin, S. Luzzatto, S. van Strien, Decay of correlations in one-dimensional dynamics, Ann. Sci. École Norm. Sup. 36 (2003), 621-646.

[6] M. Csörgö, P. Révész, A new method to prove Strassen type laws of invariance principle. I; II, Z. Wahrscheinlichkeitstheor. verw. Geb. 31 (1975), 255-259, 261-269.

[7] C. Cuny, J. Dedecker, A. Korepanov, F. Merlevède, Rates in almost sure invariance principle for slowly mixing dynamical systems, Preprint, 2018. arXiv:1801.05335. 
[8] C. Cuny, J. Dedecker, F. Merlevède, On the Komlós, Major and Tusnády strong approximation for some classes of random iterates, Stochastic Process. Appl. 128 (2018), no. 4, $1347-1385$.

[9] P. Eslami, On piecewise expanding maps, Preprint, arXiv:1711.09245 (2018).

[10] S. Gouëzel, Sharp polynomial estimates for the decay of correlations, Israel J. Math., 139 (2004), 29-65.

[11] S. Gouëzel, Decay of correlations for nonuniformly expanding systems, Bull. Soc. math. France, 134 (2006), 1-31.

[12] J. Kiefer, On the deviations in the Skorokhod-Strassen approximation scheme, Z. Wahrscheinlichkeitstheor. verw. Geb. 32 (1975), 111-131; 13 (1969), 321-332.

[13] J. Komlós, P. Major, G. Tusnády, An approximation of partial sums of independent $R V^{\prime}-s$ and the sample DF. I; II, Z. Wahrscheinlichkeitstheor. verw. Geb. 32 (1975), 111-131; 34 (1976), 34-58.

[14] A. Korepanov, Rates in almost sure invariance principle for dynamical systems with some hyperbolicity, Comm. Math. Phys. 363 (2018), 173-190.

[15] A. Korepanov, Z. Kosloff and I. Melbourne. Explicit coupling argument for nonuniformly hyperbolic transformations, Preprint, 2016. To appear in Proc. Roy. Soc. Edinburgh Sect. A.

[16] A. Korepanov, Z. Kosloff and I. Melbourne. Martingale-coboundary decomposition for families of dynamical systems, Ann. Inst. H. Poincaré Anal. Non Linéaire 35 (2018), 859-885.

[17] T. Lindvall, On Coupling of Discrete Renewal Processes, Z. Wahrscheinlichkeitstheor. verw. Geb. 48 (1979), 57-70.

[18] C. Liverani, B. Saussol, and S. Vaienti, A probabilistic approach to intermittency, Ergodic Theory Dynam. Systems, 19 (1999), 671-685.

[19] P. Major, The approximation of partial sums of independent RVs, Z. Wahrscheinlichkeitstheor. verw. Geb. 35 (1976), 213-220.

[20] I. Melbourne, M. Nicol, Almost sure invariance principle for nonuniformly hyperbolic systems, Comm. Math. Phys. 260 (2005), 131-146.

[21] F. Merlevède, E. Rio, Strong approximation for additive functionals of geometrically ergodic Markov chains, Electron. J. Probab. 20 (2015), 1-27.

[22] F. Merlevède, M. Peligrad, M. and E. Rio, A Bernstein type inequality and moderate deviations for weakly dependent sequences. Probab. Theory Related Fields 151 (2011), no. $3-4,435-474$.

[23] W. Philipp, W. Stout, Almost sure invariance principles for partial sums of weakly dependent random variables, Amer. Math. Soc. Mem. 161 (1975).

[24] E. Rio, Asymptotic theory of weakly dependent random processes. Translated from the 2000 French edition. Probability Theory and Stochastic Modelling, 80. Springer, Berlin, 2017. 
[25] A.I. Sakhanenko, Rate of convergence in the invariance principle for variables with exponential moments that are not identically distributed. (Russian) Limit theorems for sums of random variables, 4-49, Trudy Inst. Mat., 3, "Nauka" Sibirsk. Otdel., Novosibirsk, (1984).

[26] A.I. Sakhanenko, Estimates in the invariance principle in terms of truncated power moments. Sibirsk. Mat. Zh. 47 (2006), 1355-1371.

[27] Q.M. Shao, Strong approximation theorems for independent random variables and their applications. J. Multivariate Anal. 52 (1995), no. 1, 107-130.

[28] V. Strassen, An invariance principle for the law of iterated logarithm, Z. Wahrscheinlichkeitstheor. verw. Geb. 3 (1964), 211-226.

[29] A.W. van der Vaart and J.A. Wellner. Weak convergence and empirical processes. With applications to statistics. Springer Series in Statistics. Springer-Verlag, New York, 1996. MR1385671

[30] L.-S. Young, Statistical properties of dynamical systems with some hyperbolicity, Ann. of Math. 147 (1998), 585-650.

[31] L.-S. Young, Recurrence times and rates of mixing, Israel J. Math. 110 (1999), 153-188.

[32] A.Yu. Zaitsev, Gaussian approximation of convolutions in the situation when multivariate analogues of Bernsteins inequality hold, Preprint -9-84, Leningrad Branch of the Steklov Institute of Mathematics, Leningrad (1984), 54 pp. 